Potential Water-Quality Effects of Coal-Bed Methane Production Water Discharged along the Upper Tongue River, Wyoming and Montana

Scientific Investigations Report 2011-5196
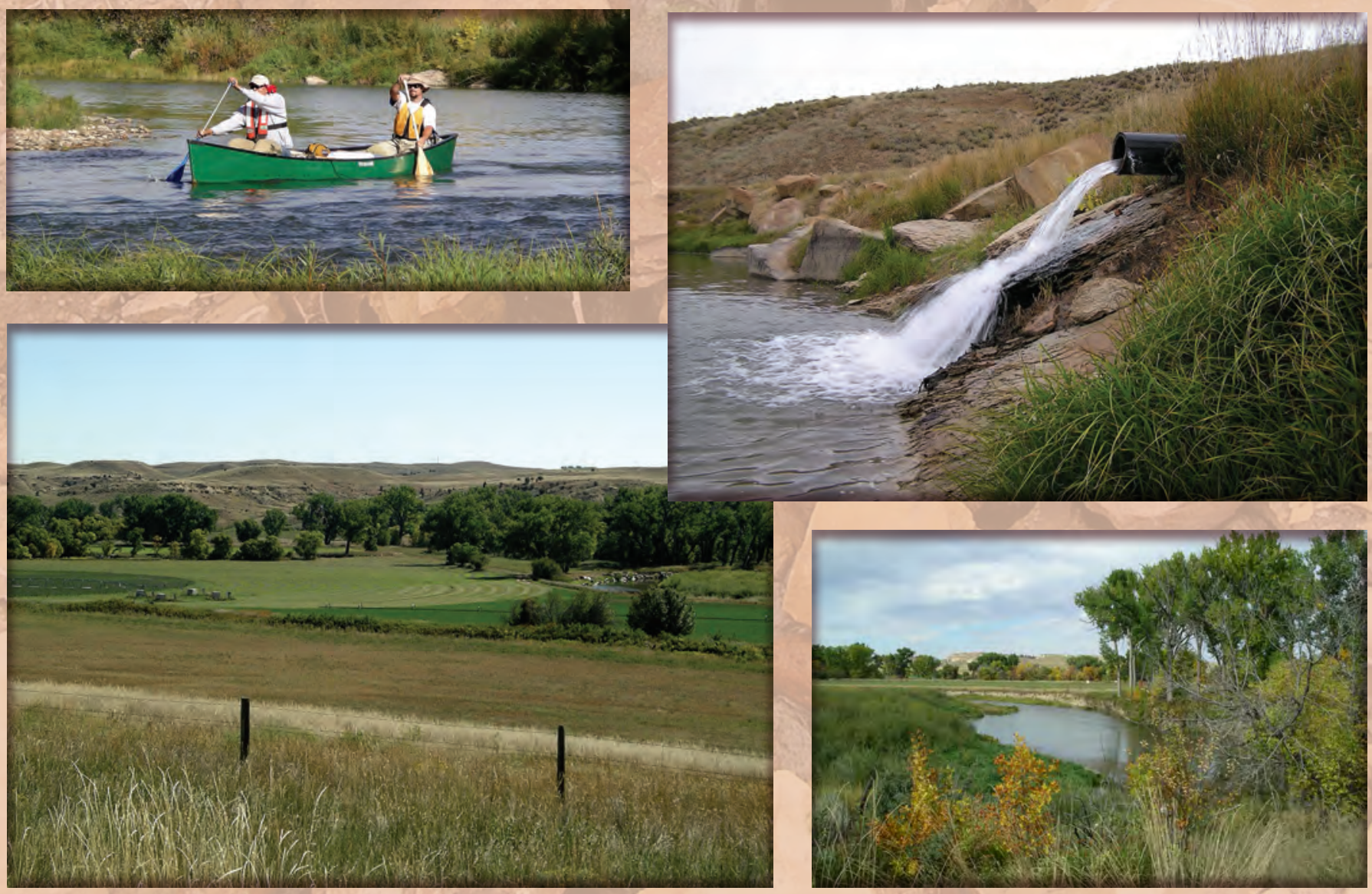

U.S. Department of the Interior

U.S. Geological Survey 
Front cover. Upper left: Aroscott Whiteman and Peter Wright (U.S. Geological Survey) canoeing the sampling reach on the Tongue River, September 2005.

Upper right: Discharge outfall (MT-0030457-015) for coal-bed methane production water near Tongue River at State line, near Decker, Mont. (gaging station 06306300), September 2005.

Lower left: Field with coal-bed methane wells near Sheridan, Wyo.

Lower right: Tongue River with coal-bed methane wells in the background, September 2005.

Background: Discharge pipes for coal-bed methane production water, Tongue River, September 2005.

Back cover. Tongue River with coal-bed methane wells in the background, September 2005.

All photographs by David A. Nimick, U.S. Geological Survey. 


\section{Potential Water-Quality Effects of Coal-Bed Methane Production Water Discharged along the Upper Tongue River, Wyoming and Montana}

By Stacy M. Kinsey and David A. Nimick

Scientific Investigations Report 2011-5196 


\section{U.S. Department of the Interior \\ KEN SALAZAR, Secretary \\ U.S. Geological Survey \\ Marcia K. McNutt, Director}

U.S. Geological Survey, Reston, Virginia: 2011

For more information on the USGS — the Federal source for science about the Earth, its natural and living resources, natural hazards, and the environment, visit http://www.usgs.gov or call 1-888-ASK-USGS.

For an overview of USGS information products, including maps, imagery, and publications, visit http://www.usgs.gov/pubprod

Any use of trade, product, or firm names is for descriptive purposes only and does not imply endorsement by the U.S. Government.

Although this report is in the public domain, permission must be secured from the individual copyright owners to reproduce any copyrighted materials contained within this report.

Suggested citation:

Kinsey, S.M., and Nimick, D.A., 2011, Potential water-quality effects of coal-bed methane production water discharged along the upper Tongue River, Wyoming and Montana: U.S. Geological Survey Scientific Investigations Report 2011-5196, 28 p. 


\section{Acknowledgments}

Thanks are extended to the individuals who assisted in this study. Particular thanks are extended to the many landowners in the study area for allowing access to their property and to Peter R. Wright, Aroscott Whiteman, and John H. Lambing (retired) of the U.S. Geological Survey for assisting with data collection. 


\section{Contents}

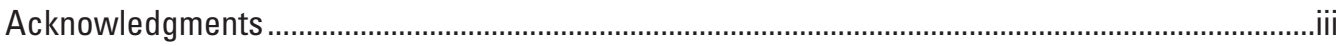

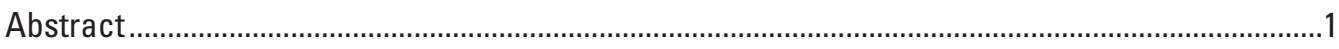

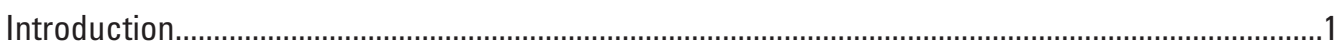

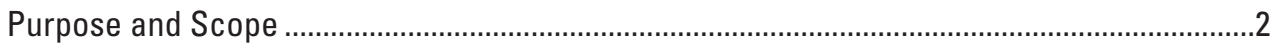

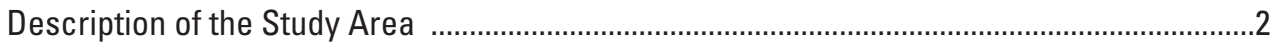

Methods of Data Collection and Analysis ................................................................................

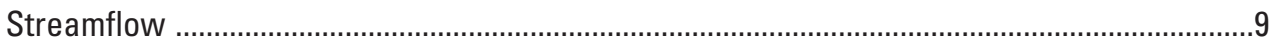

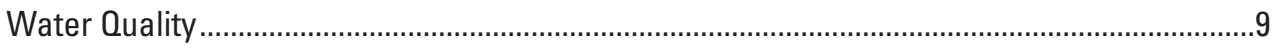

Mass-Balance and Other Calculations ................................................................................11

Specific Conductance along the Upper Tongue River ...................................................................12

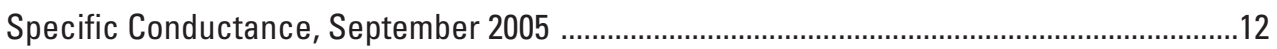

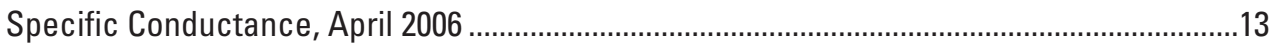

Potential Water-Quality Effects of Coal-Bed Methane Discharge along the Upper Tongue River ..........................................................................................................19

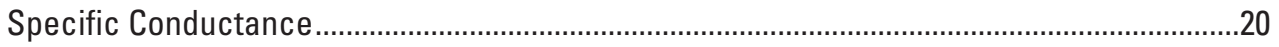

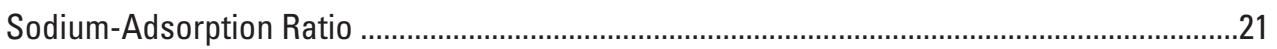

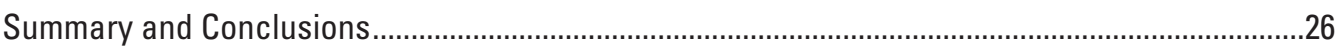

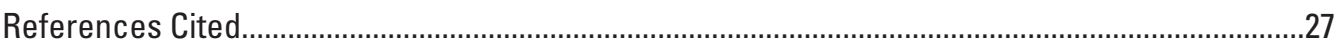

\section{Figures}

1. Maps showing locations of synoptic measurement and sampling sites along the upper Tongue River and selected tributaries, Wyoming and Montana, September 27-28, 2005

2. Maps showing locations of synoptic measurement and sampling sites along the upper Tongue River and selected tributaries, Wyoming and Montana, April 20, 2006.

3. Graphs showing continuous streamflow during September 23-30, 2005

4. Graphs showing continuous specific conductance during September 23-30, 2005

5. Graphs showing continuous streamflow during April 15-25, 2006.................................15

6. Graphs showing continuous specific conductance during April 15-25, 2006.................16

7. Graph showing specific conductance of the upper Tongue River, tributaries, and other inflows between Tongue River at Monarch, Wyo., (06299980), and State line, near Decker, Mont., (06306300), September 27-28, 2005.

8. Graph showing specific conductance of the upper Tongue River, tributaries, and other inflows between Tongue River at Monarch, Wyo. (06299980), and near the Tongue River Reservoir, Mont., April 20, 2006

9. Pie diagrams showing relative contributions of headwater and tributary inflows to the Tongue River between Monarch, Wyo., and just upstream from the Tongue River Reservoir, Mont., April 20, 2006. 
10. Graph showing relation between streamflow and measured specific conductance prior to coal-bed methane development, Tongue River at

State line, near Decker, Mont. (06306300), water years 1985-99.

11. Graph showing relation between streamflow and projected specific conductance at Tongue River near the Tongue River Reservoir, Mont., for various rates of coal-bed methane discharge.

12. Graphs showing relation between streamflow and $A$, sodium; $B$, calcium; and $C$, magnesium concentrations from water-quality samples collected prior to coal-bed methane development, Tongue River at State line, near Decker, Mont. (06306300), water years 1985-99, used to estimate sodiumadsorption ratio for various streamflows in figure 13

13. Graph showing relation between streamflow and projected sodiumadsorption ratio at Tongue River near the Tongue River Reservoir, Mont., for various rates of coal-bed methane discharge.

\section{Tables}

1. Specific conductance along the upper Tongue River, Wyoming and Montana, September 27-28, 2005

2. Physical and chemical characteristics of inflows to the upper Tongue River, Wyoming and Montana, September 27-28, 2005

3. Specific conductance along the upper Tongue River, Wyoming and Montana, April 20, 2006.

4. Physical and chemical characteristics of inflows to the upper Tongue River, Wyoming and Montana, April 20, 2006

5. State of Montana numeric standards for specific conductance and sodiumadsorption ratio

\section{Conversion Factors, Datum, Abbreviations, and Acronyms}

\begin{tabular}{lcl}
\hline \multicolumn{1}{c}{ Multiply } & By & \multicolumn{1}{c}{ To obtain } \\
\hline inch (in.) & Length & \\
inch (in.) & 2.54 & centimeter $(\mathrm{cm})$ \\
mile & 25,400 & micrometer $(\mu \mathrm{m})$ \\
\hline & 1.609 & kilometer $(\mathrm{km})$ \\
\hline cubic foot per second $\left(\mathrm{ft}^{3} / \mathrm{s}\right)$ & Flow rate & \\
gallon per minute $(\mathrm{gal} / \mathrm{min})$ & 0.02832 & cubic meter per second $\left(\mathrm{m}^{3} / \mathrm{s}\right)$ \\
\hline
\end{tabular}

Temperature in degrees Celsius $\left({ }^{\circ} \mathrm{C}\right)$ may be converted to degrees Fahrenheit $\left({ }^{\circ} \mathrm{F}\right)$ as follows:

$$
{ }^{\circ} \mathrm{F}=\left(1.8 \mathrm{x}^{\circ} \mathrm{C}\right)+32
$$

Horizontal coordinate information is referenced to the North American Datum of 1983 (NAD 83) and the North American Datum of 1927 (NAD 27). 
Water year is the 12-month period from 0 ctober 1 through September 30 of the following calendar year. The water year is designated by the calendar year in which it ends. For example, water year 2011 is the period from October 1, 2010, through September 30, 2011.

\section{Abbreviated units used in this report:}

$\mu \mathrm{S} / \mathrm{cm}$ microsiemens per centimeter at 25 degrees Celsius

$\mathrm{mg} / \mathrm{L} \quad$ milligrams per liter

\section{Acronyms used in this report:}
CBM coal-bed methane
RPD relative percent difference
SAR sodium-adsorption ratio
SC specific conductance
USGS U.S. Geological Survey 


\title{
Potential Water-Quality Effects of Coal-Bed Methane Production Water Discharged along the Upper Tongue River, Wyoming and Montana
}

\author{
By Stacy M. Kinsey and David A. Nimick
}

\section{Abstract}

Water quality in the upper Tongue River from Monarch, Wyoming, downstream to just upstream from the Tongue River Reservoir in Montana potentially could be affected by discharge of coal-bed methane (CBM) production water (hereinafter referred to as CBM discharge). CBM discharge typically contains high concentrations of sodium and other ions that could increase dissolved-solids (salt) concentrations, specific conductance (SC), and sodium-adsorption ratio (SAR) in the river. Increased inputs of sodium and other ions have the potential to alter the river's suitability for agricultural irrigation and aquatic ecosystems. Data from two large tributaries, Goose Creek and Prairie Dog Creek, indicate that these tributaries were large contributors to the increase in SC and SAR in the Tongue River. However, water-quality data were not available for most of the smaller inflows, such as small tributaries, irrigation-return flows, and CBM discharges. Thus, effects of these inflows on the water quality of the Tongue River were not well documented. Effects of these small inflows might be subtle and difficult to determine without more extensive data collection to describe spatial patterns. Therefore, synoptic water-quality sampling trips were conducted in September 2005 and April 2006 to provide a spatially detailed profile of the downstream changes in water quality in this reach of the Tongue River. The purpose of this report is to describe these downstream changes in water quality and to estimate the potential water-quality effects of CBM discharge in the upper Tongue River.

Specific conductance of the Tongue River through the study reach increased from 420 to 625 microsiemens per centimeter $(\mu \mathrm{S} / \mathrm{cm}$; or 49 percent $)$ in the downstream direction in September 2005 and from 373 to $543 \mu \mathrm{S} / \mathrm{cm}$ (46 percent) in April 2006. Large increases (12 to 24 percent) were measured immediately downstream from Goose Creek and Prairie Dog Creek during both sampling trips. Increases attributed to direct CBM discharges were smaller. In September 2005, the SC of 12 measured CBM discharges ranged from 1,750 to $2,440 \mu \mathrm{S} / \mathrm{cm}$, and the combined discharges increased SC in the river by an estimated 4.5 percent. In April 2006, the
SC of eight measured CBM discharges ranged from 1,720 to $2,070 \mu \mathrm{S} / \mathrm{cm}$; the largest of these discharges likely increased $\mathrm{SC}$ in the river by 5.8 percent.

Estimates of potential effects of the CBM discharges on the SC of the Tongue River near the Tongue River Reservoir were calculated using a two-step process involving linear regression and mass-balance calculations for a range of streamflow and CBM-discharge conditions. Potential effects from CBM discharges are larger increases of SC and SAR at lower flows than at higher flows and relative increases that are substantially smaller for SC than for SAR. For example, if the streamflow was 100 cubic feet per second $\left(\mathrm{ft}^{3} / \mathrm{s}\right)$ in the Tongue River near the Tongue River Reservoir and CBM discharge ranged from 1,250 to 5,000 gallons per minute, the projected increases would range from 4.4 to 16 percent for SC and from 39 to 151 percent for SAR. In comparison, if the streamflow was $600 \mathrm{ft}^{3} / \mathrm{s}$, the projected increases would range from 2.2 to 8.4 percent for SC and from 21 to 79 percent for SAR. This analysis of potential water-quality effects on the SC and SAR of the Tongue River in the study area assumes that the quantity and quality of water flowing into the study reach at the time of this study was the same as during the period before CBM development (data from water years 1985-99).

\section{Introduction}

Coal-bed methane (CBM) development began in the Tongue River watershed in about 1999 and currently (2011) occurs primarily near the Montana-Wyoming State line. Groundwater produced during CBM development typically has high concentrations of sodium and low concentrations of calcium and magnesium (Rice and others, 2000; Van Voast, 2003), resulting in a high sodium-adsorption ratio (SAR). This production water (hereinafter referred to as CBM discharge) commonly is discharged to surface-water drainages or constructed reservoirs (figs. 1 and 2). Although some CBM development is downstream from the Tongue River Reservoir, most of the CBM discharge outfalls are upstream from the reservoir. $\mathrm{CBM}$ discharge into the Tongue River or its tributaries has the 
potential to increase the salinity and SAR of water in the river, thereby decreasing the suitability of Tongue River water for irrigation because water having increased SAR can deteriorate soil structure (U.S. Department of Agriculture, 1995). Because agricultural irrigation is the predominant consumptive water use in the Tongue River watershed, there is concern about potential degradation of water quality from CBM discharges. An additional concern is the potential risk to aquatic ecosystems if water quality is altered in the streams receiving CBM discharge.

Specific conductance (SC) is a characteristic of water that can be related to the dissolved-solids concentration, which is referred to as salinity, or salt content (Hem, 1985). Typically, the SC of the Tongue River increases as the river flows downstream from its headwaters to the Tongue River Reservoir through areas where CBM has been developed. For example, the mean daily mean SC for the March to October season during 2004-06 was $380 \mu \mathrm{S} / \mathrm{cm}$ at Tongue River at Monarch, Wyo. (gaging station 06299980, hereinafter referred to as Tongue River at Monarch), which is upstream from CBM-development areas. At Tongue River at State line, near Decker, Mont. (gaging station 06306300, hereinafter referred to as Tongue River at State line), which is downstream from most CBM-development areas, the mean daily mean SC for this period was $627 \mu \mathrm{S} / \mathrm{cm}$ (Berkas and others, 2005, 2006; U.S. Geological Survey, 2007d). Similarly, the mean daily mean estimated SAR for March to October during 2004-06 was higher downstream at Tongue River at State line (0.80) than upstream at Tongue River at Monarch (0.33). Waterquality data for Goose Creek near Acme, Wyo. (gaging station 06305700, hereinafter referred to as Goose Creek near Acme) and Prairie Dog Creek near Acme, Wyo. (gaging station 06306250, hereinafter referred to as Prairie Dog Creek near Acme) indicate that these tributaries were a large contributor to the increase in SC and SAR in the Tongue River (U.S. Geological Survey, 2007a, b). However, water-quality data were not available for most of the smaller inflows, such as small tributaries, irrigation-return flows, and CBM discharges. Thus, effects of these inflows on the water quality of the Tongue River were not well documented. Effects of these small inflows, either individually or collectively, might be subtle and difficult to determine without more extensive data collection to describe spatial patterns. Therefore, for this study, the U.S. Geological Survey (USGS) collected synoptic water-quality samples along the upper Tongue River (between Monarch, Wyo., and the Tongue River Reservoir) on two occasions (September 2005 and April 2006) to examine downstream changes in water quality.

\section{Purpose and Scope}

The purpose of this report is to describe the downstream changes in water quality along the upper Tongue River between Monarch, Wyoming, and the Tongue River Reservoir in Montana as indicated by the results of two synoptic sampling trips and to estimate the potential water-quality effects of CBM discharge in the upper Tongue River. Waterquality data were collected during synoptic sampling trips on September 27-28, 2005, and April 20, 2006, to provide a spatially detailed profile of SC along the length of the upper Tongue River. During each synoptic sampling trip, SC of the river water was measured at multiple locations through the reach, particularly upstream and downstream from tributaries, irrigation returns, and $\mathrm{CBM}$ discharges permitted at the time of sampling (figs. 1 and 2). In addition, SC of most inflows was measured, and water-quality samples were collected from selected inflows for analysis of SAR. These data, in conjunction with other water-quality and continuous SC data collected at the four USGS gaging stations upstream from the Tongue River Reservoir, were used to estimate the load of salt contributed from various sources upstream from the reservoir.

During the September 2005 synoptic sampling trip, $23 \mathrm{SC}$ measurements were made from the main stem of the Tongue River and $17 \mathrm{SC}$ measurements were made from the inflows. Seven water-quality samples were collected from the inflows. Sampled inflows were Ash Creek, Youngs Creek, and 5 of the $15 \mathrm{CBM}$ discharges that were permitted at the time of sampling.

During the April 2006 synoptic sampling trip, 46 SC measurements were made from the main stem of the Tongue River and $12 \mathrm{SC}$ measurements were made from the inflows. Four water-quality samples were collected from the inflows. Sampled inflows were Youngs Creek and 3 of the 15 CBM discharges that were permitted at the time of sampling.

The potential effects on water quality in the upper Tongue River also were assessed by comparing historical SC and SAR values estimated for the period prior to CBM development with $\mathrm{SC}$ and $\mathrm{SAR}$ values projected for various rates of $\mathrm{CBM}$ discharge. The projected increases in SC and SAR resulting from CBM discharges to the river were estimated by using linear regression and mass-balance calculations for a range of streamflows [50-3,000 cubic feet per second $\left.\left(\mathrm{ft}^{3} / \mathrm{s}\right)\right]$ representative of most historical flow conditions.

\section{Description of the Study Area}

The Tongue River is divided into three reaches by main-stem impoundments. The upper reach extends from the headwaters in the Bighorn Mountains to the Tongue River Reservoir and includes the reach of the upper Tongue River investigated for this study. The middle reach extends from Tongue River Dam to the T\&Y Diversion Dam, which is just upstream from Pumpkin Creek. The lower reach extends from the T\&Y Diversion Dam to the confluence with the Yellowstone River at Miles City. The study area is a reach of the upper Tongue River extending from the gaging station at Monarch (gaging station 06299980) downstream to the Otter Road bridge, which is just upstream from the Tongue River Reservoir (fig. 1). Tongue River at Monarch is upstream from all CBM development within the watershed. 


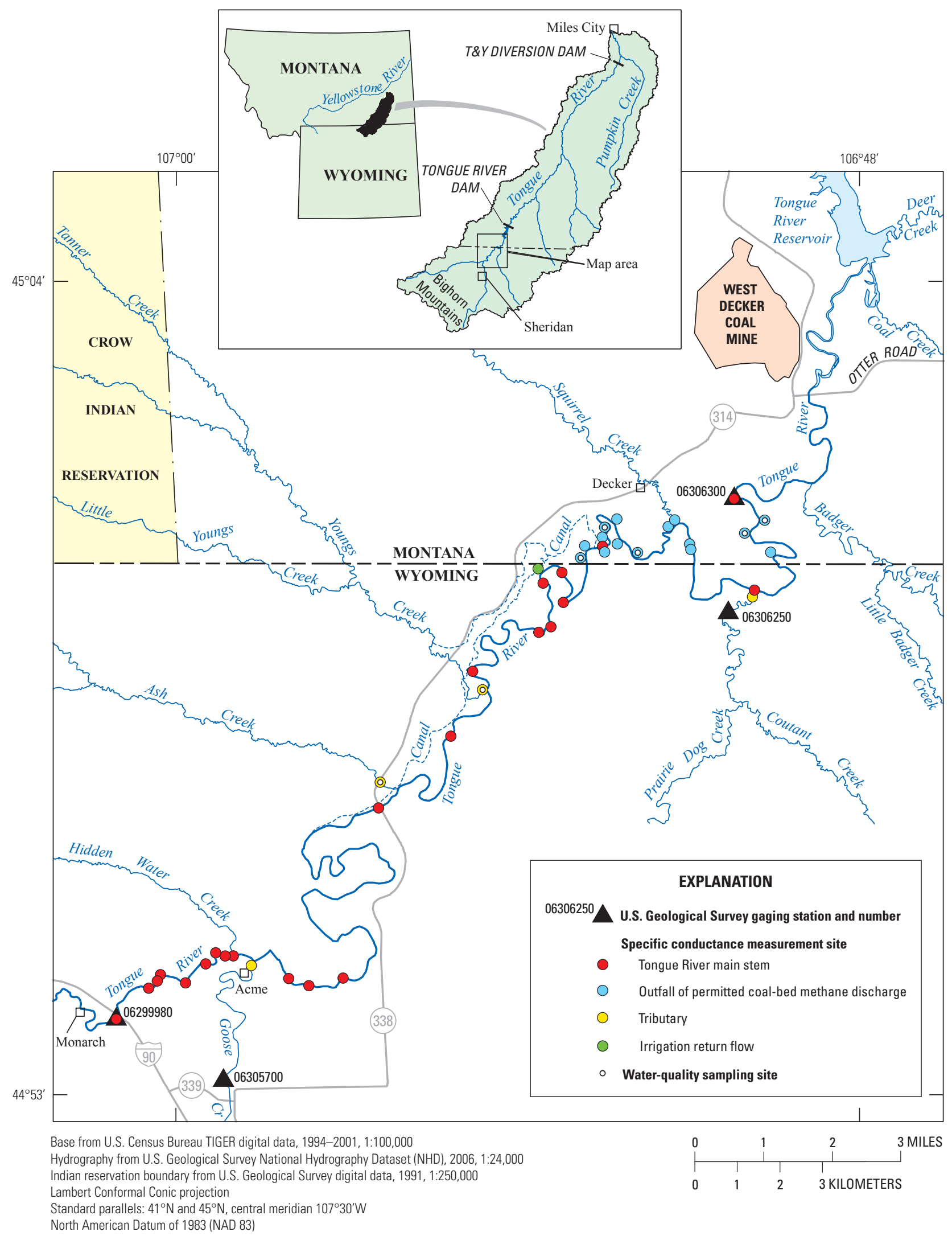

Figure 1. Locations of synoptic measurement and sampling sites along the upper Tongue River and selected tributaries, Wyoming and Montana, September 27-28, 2005. 


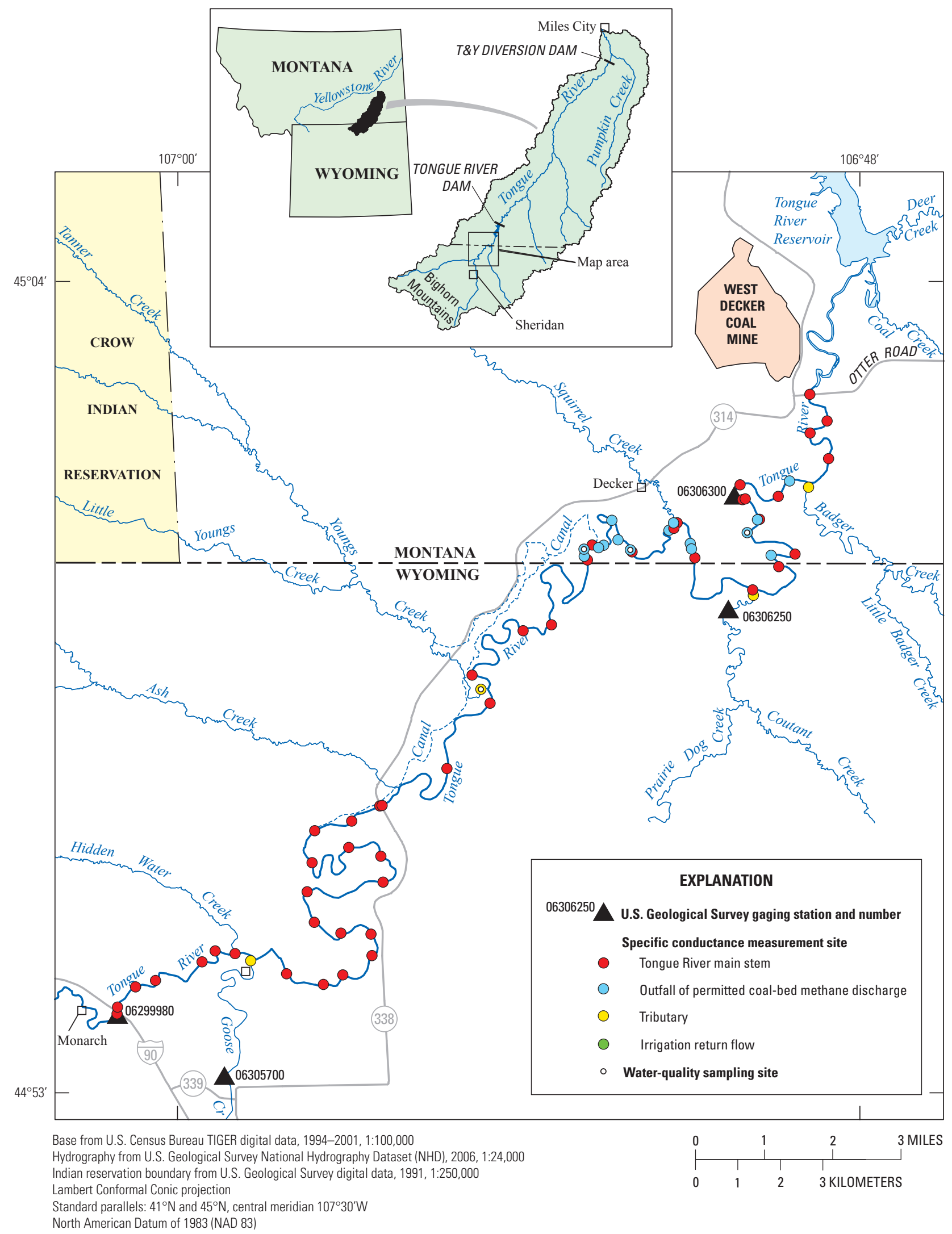

Figure 2. Locations of synoptic measurement and sampling sites along the upper Tongue River and selected tributaries, Wyoming and Montana, April 20, 2006. 
Two major tributaries enter the upper Tongue River in the study area: Goose Creek and Prairie Dog Creek (fig. 1). Streamflow for these tributaries varies both seasonally and annually. Annual mean streamflow for Goose Creek near Acme (gaging station 06305700) ranged from about 50 to $303 \mathrm{ft}^{3} / \mathrm{s}$ during water years 1984-2006 (U.S. Geological Survey, 2007a), and the mean annual streamflow was $144 \mathrm{ft}^{3} / \mathrm{s}$. Goose Creek receives discharge from the wastewatertreatment facility in Sheridan, Wyo. Annual mean streamflow for Prairie Dog Creek near Acme (gaging station 06306250) ranged from about 14 to $73 \mathrm{ft}^{3} / \mathrm{s}$ during water years 1971-2006 (U.S. Geological Survey, 2007b), and the mean annual streamflow was $36 \mathrm{ft}^{3} / \mathrm{s}$. More than 1,000 CBM wells have been drilled in the Prairie Dog Creek drainage since 2000 (Wyoming Oil and Gas Commission, 2008b).

Four small tributaries enter the upper Tongue River in the study area (Ash, Youngs, Squirrel, and Badger Creeks; fig. 1). Hundreds of CBM wells have been drilled in the drainages of these four tributaries since 2000 (Wyoming Oil and Gas Commission, 2008a, b, c), although there are no permits to dispose of CBM discharge into these tributaries (Tom Reid, Montana Department of Environmental Quality, written commun., 2008).

The study reach had 15 direct CBM discharge outfalls; all were located in Montana and were permitted by the State of Montana at the time of the synoptic sampling trips (Montana Department of Environmental Quality, written commun., 2006; figs. 1 and 2). Twelve of the CBM discharge outfalls were upstream from Prairie Dog Creek, and the others were downstream from Prairie Dog Creek. During the April 2006 synoptic sampling trip, outfall MT-0030457-003 could not be located, therefore, 11 of the 15 CBM discharge outfalls were upstream from Prairie Dog Creek (fig. 2). The permit (MT-0030457-003) authorized a maximum combined CBM discharge of 2,500 gallons per minute (gal $/ \mathrm{min})$, or $5.57 \mathrm{ft}^{3} / \mathrm{s}$, from the 15 outfalls to the Tongue River. The actual CBM discharge measured during both synoptic sampling trips was less than the permitted discharge because field measurement of streamflow at some outfalls was not practicable and because the amount of CBM discharge can fluctuate in response to the amount of water being removed from the aquifer in order to extract the methane gas (Keith and others, 2003). Although the number of outfalls was the same for both synoptic sampling trips, outfall MT-0030457-15 was relocated to a new site downstream from Tongue River at State line (gaging station 06306300) and renamed MT-0030457-16 after the September 2005 synoptic sampling trip (Montana Department of Environmental Quality, written commun., 2006). In 2010, the Montana
Supreme Court voided the permit for the 15 direct CBM discharges in the study reach (Montana Supreme Court, 2010).

\section{Methods of Data Collection and Analysis}

Two synoptic sampling trips were designed to (1) determine the location of inflows by canoeing along the Tongue River in the reach upstream from the Tongue River Reservoir and (2) spatially delineate and quantify downstream changes in SC. In September 2005, the synoptic sampling trip extended from Tongue River at Monarch (gaging station 06299980) to Tongue River at State line (gaging station 06306300). The downstream part of the trip (river mile 15.54 to 30.57) was conducted on September 27; the upstream part (river mile 0.00 to 12.94) was conducted on September 28 (fig. 1; tables 1 and 2). The part of the river between river mile 4.54 and 12.94 was not visited or sampled because information from topographic maps and descriptions of CBM permit locations (Montana Department of Environmental Quality, written commun., 2006) indicated that this part of the river lacked inflows that could affect the SC of the Tongue River.

In April 2006, the synoptic sampling trip extended from Tongue River at Monarch to the county bridge on Otter Road, which is just upstream from the Tongue River Reservoir. This sampling trip covered a longer reach than the trip in September 2005 so that Badger Creek and a new CBM discharge outfall downstream from Tongue River at State line could be included in the investigation. The entire trip was conducted in 1 day (April 20) by utilizing two study teams. The first team covered river mile 0.00 to 12.50 and then river mile 30.57 to 34.51 , while the second team covered river mile 12.50 to 30.57 (fig. 2; tables 3 and 4). During this synoptic sampling trip, the reach from river mile 4.54 and 12.94 was visited and no inflows to the main stem were found.

Tongue River at Monarch, Tongue River at State line, Goose Creek near Acme, and Prairie Dog Creek near Acme were sampled periodically throughout 2005 and 2006 as part of ongoing monitoring activities; therefore, these sites were not sampled during either synoptic sampling trip. Most tributaries were sampled at their mouth during the synoptic sampling trips (figs. 1 and 2). CBM discharge outfalls were selected for sampling on the basis of amount of flow, location, and visibility from the river. Very small or diffuse CBM discharges were not sampled. 
Table 1. Specific conductance along the upper Tongue River, Wyoming and Montana, September 27-28, 2005.

[Sites are listed in downstream order. Abbreviation: $\mathrm{ft}^{3} / \mathrm{s}$, cubic feet per second; $\mu \mathrm{S} / \mathrm{cm}$, microsiemens per centimeter at 25 degrees Celsius; SAR, sodium-adsorption ratio. Symbol: --, no data]

\begin{tabular}{|c|c|c|c|c|c|c|c|c|}
\hline Latitude $^{1}$ & Longitude $^{1}$ & $\begin{array}{l}\text { River } \\
\text { mile }^{2}\end{array}$ & Date & Time & $\begin{array}{c}\text { Specific } \\
\text { conductance } \\
(\mu \mathrm{S} / \mathrm{cm})\end{array}$ & $\begin{array}{l}\text { Streamflow } \\
\quad\left(\mathrm{ft}^{3} / \mathrm{s}\right)\end{array}$ & $S A R^{3}$ & Remarks \\
\hline $44^{\circ} 54^{\prime} 8.20^{\prime \prime}$ & $107^{\circ} 1.0^{\prime} 12.1^{\prime \prime}$ & 0.00 & $9 / 28 / 2005$ & 0830 & 420 & 74 & 0.36 & $\begin{array}{l}\text { Tongue River at Monarch, Wyo. (gaging station 06299980). Continuous } \\
\text { streamflow and specific conductance data recorded. }\end{array}$ \\
\hline $44^{\circ} 54^{\prime} 25.3^{\prime \prime}$ & $107^{\circ} 0.0^{\prime} 41.6^{\prime \prime}$ & .64 & $9 / 28 / 2005$ & 0845 & 419 & -- & -- & \\
\hline $44^{\circ} 54^{\prime} 29.2^{\prime \prime}$ & $107^{\circ} 0.0^{\prime} 30.5^{\prime \prime}$ & .84 & $9 / 28 / 2005$ & 0850 & 419 & -- & -- & \\
\hline $44^{\circ} 54^{\prime} 34.0^{\prime \prime}$ & $107^{\circ} 0.0^{\prime} 29.4^{\prime \prime}$ & 1.04 & $9 / 28 / 2005$ & 0857 & 419 & -- & -- & \\
\hline $44^{\circ} 54^{\prime} 27.6^{\prime \prime}$ & $107^{\circ} 0.0^{\prime} 2.3^{\prime \prime}$ & 1.40 & $9 / 28 / 2005$ & 0908 & 419 & -- & -- & \\
\hline $44^{\circ} 54^{\prime} 43.0^{\prime \prime}$ & $106^{\circ} 59^{\prime} 40.3^{\prime \prime}$ & 1.84 & $9 / 28 / 2005$ & 0917 & 420 & -- & -- & \\
\hline $44^{\circ} 54^{\prime} 51.6^{\prime \prime}$ & $106^{\circ} 59^{\prime} 29.8^{\prime \prime}$ & 2.24 & $9 / 28 / 2005$ & 0925 & 420 & -- & -- & \\
\hline $44^{\circ} 54^{\prime} 48.6^{\prime \prime}$ & $106^{\circ} 59^{\prime} 19.7^{\prime \prime}$ & 2.44 & $9 / 28 / 2005$ & 0936 & 420 & -- & -- & \\
\hline $44^{\circ} 54^{\prime} 47.6^{\prime \prime}$ & $106^{\circ} 59^{\prime} 10.9^{\prime \prime}$ & 2.54 & $9 / 28 / 2005$ & 0938 & 420 & -- & -- & Upstream from Goose Creek. \\
\hline $44^{\circ} 54^{\prime} 33.6^{\prime \prime}$ & $106^{\circ} 58^{\prime} 11.2^{\prime \prime}$ & 3.64 & $9 / 28 / 2005$ & 1016 & 520 & -- & -- & Downstream from Goose Creek. \\
\hline $44^{\circ} 54^{\prime} 26.4^{\prime \prime}$ & $106^{\circ} 57^{\prime} 50.2^{\prime \prime}$ & 3.94 & $9 / 28 / 2005$ & 1024 & 520 & -- & -- & \\
\hline $44^{\circ} 54^{\prime} 28.7^{\prime \prime}$ & $106^{\circ} 57^{\prime} 13.5^{\prime \prime}$ & 4.54 & $9 / 28 / 2005$ & 1050 & 520 & -- & -- & \\
\hline $44^{\circ} 56^{\prime} 44.0^{\prime \prime}$ & $106^{\circ} 56^{\prime} 35.0^{\prime \prime}$ & 12.94 & $9 / 28 / 2005$ & 1130 & 522 & -- & -- & Upstream from Ash Creek. \\
\hline $44^{\circ} 57^{\prime} 33.5^{\prime \prime}$ & $106^{\circ} 55^{\prime} 16.2^{\prime \prime}$ & 15.54 & $9 / 27 / 2005$ & 0941 & 539 & -- & -- & Downstream from Ash Creek. \\
\hline $44^{\circ} 58^{\prime} 24.7^{\prime \prime}$ & $106^{\circ} 54^{\prime} 50.7^{\prime \prime}$ & 17.04 & $9 / 27 / 2005$ & 1100 & 543 & -- & -- & Downstream from Youngs Creek. \\
\hline $44^{\circ} 58^{\prime} 55.3^{\prime \prime}$ & $106^{\circ} 53^{\prime} 40.7^{\prime \prime}$ & 19.34 & $9 / 27 / 2005$ & 1140 & 544 & -- & -- & \\
\hline $44^{\circ} 58^{\prime} 56.1^{\prime \prime}$ & $106^{\circ} 53^{\prime} 25.0^{\prime \prime}$ & 19.74 & $9 / 27 / 2005$ & 1200 & 542 & -- & -- & \\
\hline $44^{\circ} 59^{\prime} 31.6^{\prime \prime}$ & $106^{\circ} 53^{\prime} 35.9^{\prime \prime}$ & 20.48 & $9 / 27 / 2005$ & 1215 & 542 & -- & -- & \\
\hline $44^{\circ} 59^{\prime} 41.4^{\prime \prime}$ & $106^{\circ} 53^{\prime} 14.7^{\prime \prime}$ & 20.94 & $9 / 27 / 2005$ & 1235 & 541 & -- & -- & Downstream from irrigation return flow. \\
\hline $44^{\circ} 59^{\prime} 16.7^{\prime \prime}$ & $106^{\circ} 53^{\prime} 13.7^{\prime \prime}$ & 21.54 & $9 / 27 / 2005$ & 1253 & 540 & -- & -- & $\begin{array}{l}\text { Upstream from all Montana coal-bed } \\
\text { methane discharge outfalls. }\end{array}$ \\
\hline $44^{\circ} 59^{\prime} 56.5^{\prime \prime}$ & $106^{\circ} 52^{\prime} 31.9^{\prime \prime}$ & 22.84 & $9 / 27 / 2005$ & 1400 & 539 & -- & -- & \\
\hline $44^{\circ} 59^{\prime} 24.0^{\prime \prime}$ & $106^{\circ} 49^{\prime} 49.2^{\prime \prime}$ & 27.94 & $9 / 27 / 2005$ & 1645 & 544 & -- & -- & Upstream from Prairie Dog Creek. \\
\hline $45^{\circ} 00^{\prime} 32.0^{\prime \prime}$ & $106^{\circ} 50^{\prime} 08.0^{\prime \prime}$ & 30.57 & $9 / 27 / 2005$ & 1830 & 625 & 195 & .76 & $\begin{array}{l}\text { Tongue River at State line, near Decker, Mont. (gaging station 06306300). } \\
\text { Continuous streamflow and specific conductance data recorded. }\end{array}$ \\
\hline
\end{tabular}

${ }^{1}$ Latitude and longitude, in degrees, minutes and seconds, are referenced to the North American Datum of 1983 (NAD 83).

${ }^{2}$ Distance downstream from Tongue River at Monarch (gaging station 06299980).

${ }^{3}$ Estimated from real-time specific conductance by using linear-regression methods (Cannon and others, 2007). 
Table 2. Physical and chemical characteristics of inflows to the upper Tongue River, Wyoming and Montana, September 27-28, 2005.

[Site names for permitted coal-bed methane discharges correspond to names designated by the Montana Department of Environmental Quality (for example, MT-0030457-009). Sites are listed in downstream order. Sodium-adsoprtion ratio was calculated from laboratory analytical data unless otherwise noted. Site type: C, permitted coal-bed methane discharge; I, irrigation return flow; T, tributary. Abbreviations: e, estimated; $\mathrm{ft}^{3} / \mathrm{s}$, cubic feet per second; $\mu \mathrm{S} / \mathrm{cm}$, microsiemens per centimeter at 25 degrees Celsius; $\mathrm{mg} / \mathrm{L}$, milligrams per liter; SAR, sodium-adsorption ratio. Symbols: <, less than; --, no data]

\begin{tabular}{|c|c|c|c|c|c|c|c|c|c|c|c|c|c|}
\hline Site name & $\begin{array}{l}\text { U.S. Geological } \\
\text { Survey gaging } \\
\text { station or } \\
\text { miscellaneous } \\
\text { measurement } \\
\text { number }\end{array}$ & Latitude $^{1}$ & Longitude $^{1}$ & $\begin{array}{l}\text { River } \\
\text { mile }^{2}\end{array}$ & $\begin{array}{l}\text { Site } \\
\text { type }\end{array}$ & Date & Time & $\begin{array}{c}\text { Stream- } \\
\text { flow } \\
\left(\mathrm{ft}^{3} / \mathrm{s}\right)\end{array}$ & $\begin{array}{c}\text { Specific } \\
\text { conduc- } \\
\text { tance } \\
(\mu \mathrm{S} / \mathrm{cm})\end{array}$ & $\begin{array}{l}\text { Dissolved } \\
\text { sodium } \\
\text { (mg/L) }\end{array}$ & $\begin{array}{l}\text { Dissolved } \\
\text { calcium } \\
\text { (mg/L) }\end{array}$ & $\begin{array}{l}\text { Dissolved } \\
\text { magnesium } \\
\text { (mg/L) }\end{array}$ & SAR \\
\hline $\begin{array}{l}\text { Goose Creek near } \\
\text { Acme, Wyo. }\end{array}$ & 06305700 & $44^{\circ} 54^{\prime} 44.6^{\prime \prime}$ & $106^{\circ} 58^{\prime} 45.3^{\prime \prime}$ & 2.81 & $\mathrm{~T}$ & $9 / 28 / 2005$ & 1000 & 87 & 612 & -- & -- & -- & ${ }^{3} 0.63$ \\
\hline $\begin{array}{l}\text { Ash Creek near } \\
\text { mouth }\end{array}$ & 445700106563101 & $44^{\circ} 57^{\prime} 00.0^{\prime \prime}$ & $106^{\circ} 56^{\prime} 31.0^{\prime \prime}$ & 13.07 & $\mathrm{~T}$ & $9 / 28 / 2005$ & 1135 & .10 & 1,700 & 95.0 & 123 & 113 & 1.0 \\
\hline $\begin{array}{l}\text { Youngs Creek at } \\
\text { mouth }\end{array}$ & 445817106544601 & $44^{\circ} 58^{\prime} 17.0^{\prime \prime}$ & $106^{\circ} 54^{\prime} 46.2^{\prime \prime}$ & 16.52 & $\mathrm{~T}$ & $9 / 27 / 2005$ & 1030 & .27 & 1,260 & 54.5 & 83.8 & 99.5 & 1.0 \\
\hline $\begin{array}{l}\text { Unnamed irrigation } \\
\text { return flow }\end{array}$ & & $44^{\circ} 59^{\prime} 39.1^{\prime \prime}$ & $106^{\circ} 53^{\prime} 37.2^{\prime \prime}$ & 20.60 & I & $9 / 27 / 2005$ & 1230 & -- & 530 & -- & -- & -- & -- \\
\hline MT-0030457-009 & 445949106524801 & $44^{\circ} 59^{\prime} 48.9^{\prime \prime}$ & $106^{\circ} 52^{\prime} 48.4^{\prime \prime}$ & 22.17 & $\mathrm{C}$ & $9 / 27 / 2005$ & 1315 & .26 & 2,050 & 464 & 5.89 & 2.19 & 42 \\
\hline MT-0030457-008 & & $44^{\circ} 59^{\prime} 55.2^{\prime \prime}$ & $106^{\circ} 52^{\prime} 48.0^{\prime \prime}$ & 22.27 & $\mathrm{C}$ & $9 / 27 / 2005$ & 1340 & .002 & 2,160 & -- & -- & -- & -- \\
\hline MT-0030457-002 & & $44^{\circ} 59^{\prime} 56.5^{\prime \prime}$ & $106^{\circ} 52^{\prime} 31.9^{\prime \prime}$ & 22.47 & $\mathrm{C}$ & $9 / 27 / 2005$ & 1354 & ${ }^{4}<.01$ & $5--$ & -- & -- & -- & -- \\
\hline MT-0030457-003 & & $45^{\circ} 00^{\prime} 06.8^{\prime \prime}$ & $106^{\circ} 52^{\prime} 27.2^{\prime \prime}$ & 22.73 & $\mathrm{C}$ & $9 / 27 / 2005$ & 1405 & Dry & -- & -- & -- & -- & -- \\
\hline MT-0030457-004 & 450011106522501 & $45^{\circ} 00^{\prime} 10.9^{\prime \prime}$ & $106^{\circ} 52^{\prime} 25.2^{\prime \prime}$ & 22.77 & $\mathrm{C}$ & $9 / 27 / 2005$ & 1413 & .034 & 1,980 & 467 & 3.70 & 1.31 & 53 \\
\hline MT-0030457-001 & & $45^{\circ} 00^{\prime} 17.3^{\prime \prime}$ & $106^{\circ} 52^{\prime} 17.9^{\prime \prime}$ & 23.37 & $\mathrm{C}$ & $9 / 27 / 2005$ & 1440 & .004 & 1,880 & -- & -- & -- & -- \\
\hline MT-0030457-010 & & $45^{\circ} 00^{\prime} 02.7^{\prime \prime}$ & $106^{\circ} 52^{\prime} 11.2^{\prime \prime}$ & 23.67 & $\mathrm{C}$ & $9 / 27 / 2005$ & 1450 & .009 & 2,040 & -- & -- & -- & -- \\
\hline MT-0030457-005 & 445955106515801 & $44^{\circ} 59^{\prime} 54.8^{\prime \prime}$ & $106^{\circ} 51^{\prime} 57.6^{\prime \prime}$ & 23.87 & $\mathrm{C}$ & $9 / 27 / 2005$ & 1505 & .022 & 1,750 & 417 & 3.65 & 1.25 & 48 \\
\hline MT-0030457-006 & & $45^{\circ} 00^{\prime} 09.7^{\prime \prime}$ & $106^{\circ} 51^{\prime} 16.2^{\prime \prime}$ & 24.97 & $\mathrm{C}$ & $9 / 27 / 2005$ & 1535 & .21 & 1,960 & -- & -- & -- & -- \\
\hline MT-0030457-007 & & $45^{\circ} 00^{\prime} 15.8^{\prime \prime}$ & $106^{\circ} 51^{\prime} 10.8^{\prime \prime}$ & 25.07 & $\mathrm{C}$ & $9 / 27 / 2005$ & 1547 & .16 & 2,000 & -- & -- & -- & -- \\
\hline $\begin{array}{l}\text { Squirrel Creek at } \\
\text { mouth }\end{array}$ & & $45^{\circ} 00^{\prime} 15.5^{\prime \prime}$ & $106^{\circ} 51^{\prime} 04.2^{\prime}$ & 25.12 & $\mathrm{~T}$ & $9 / 27 / 2005$ & 1600 & Dry & -- & -- & -- & -- & -- \\
\hline MT-0030457-011 & & $44^{\circ} 59^{\prime} 55.1^{\prime \prime}$ & $106^{\circ} 50^{\prime} 52.0^{\prime \prime}$ & 25.47 & $\mathrm{C}$ & $9 / 27 / 2005$ & 1615 & Dry & -- & -- & -- & -- & -- \\
\hline MT-0030457-014 & & $44^{\circ} 59^{\prime} 53.5^{\prime \prime}$ & $106^{\circ} 50^{\prime} 53.3^{\prime \prime}$ & 25.62 & $\mathrm{C}$ & $9 / 27 / 2005$ & 1620 & .33 & 1,830 & -- & -- & -- & -- \\
\hline $\begin{array}{l}\text { Prairie Dog Creek } \\
\text { near Acme, Wyo. }\end{array}$ & 06306250 & $44^{\circ} 59^{\prime} 24^{\prime \prime}$ & $106^{\circ} 49^{\prime} 49.2^{\prime \prime}$ & 27.99 & $\mathrm{~T}$ & $9 / 27 / 2005$ & 1650 & 31 & 1,034 & -- & -- & -- & ${ }^{3} .87$ \\
\hline MT-0030457-012 & & $44^{\circ} 59^{\prime} 49.6^{\prime \prime}$ & $106^{\circ} 49^{\prime} 26.0^{\prime \prime}$ & 28.71 & $\mathrm{C}$ & $9 / 27 / 2005$ & 1715 & .056 & 1,990 & -- & -- & -- & -- \\
\hline MT-0030457-013 & 450007106495201 & $45^{\circ} 00^{\prime} 07.2^{\prime \prime}$ & $106^{\circ} 49^{\prime} 51.7^{\prime \prime}$ & 29.91 & $\mathrm{C}$ & $9 / 27 / 2005$ & 1735 & .035 & 1,970 & 488 & 3.55 & 1.28 & 57 \\
\hline MT-0030457-015 & 450017106494001 & $45^{\circ} 00^{\prime} 17.0^{\prime \prime}$ & $106^{\circ} 49^{\prime \prime} 40.0^{\prime \prime}$ & 30.17 & $\mathrm{C}$ & $9 / 27 / 2005$ & 1750 & $\mathrm{e} 2.0$ & 2,440 & 581 & 4.59 & 1.84 & 58 \\
\hline
\end{tabular}


Table 3. Specific conductance along the upper Tongue River, Wyoming and Montana, April 20, 2006.

[Sites are listed in downstream order. Shaded portion highlights the time difference in readings. Abbreviation: $\mathrm{ft}^{3} / \mathrm{s}$, cubic feet per second; $\mu \mathrm{S} / \mathrm{cm}, \mathrm{microsiemens}$ per centimeter at 25 degrees Celsius; SAR, sodium-adsorption ratio. Symbol: --, no data]

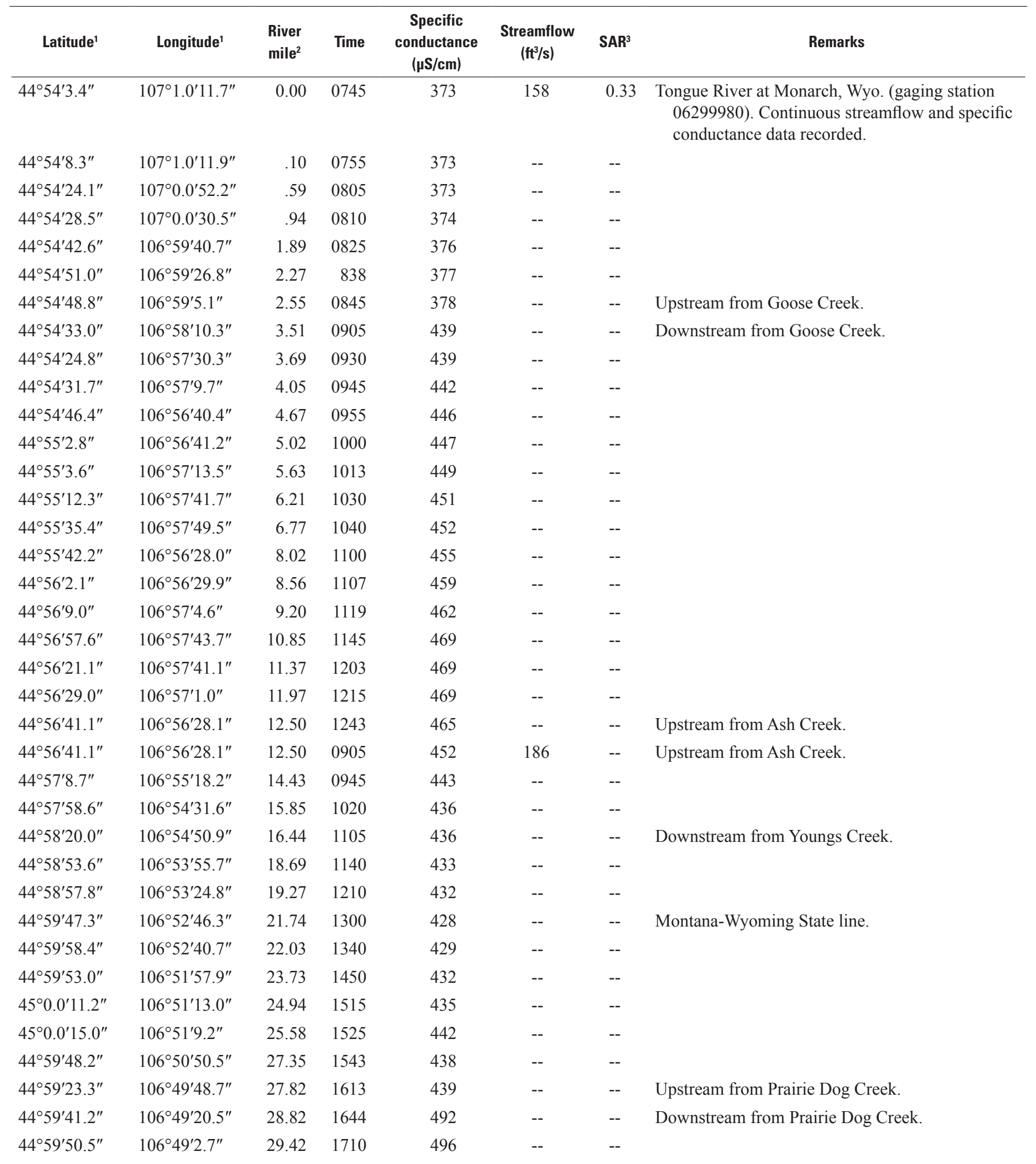


Table 3. Specific conductance along the upper Tongue River, Wyoming and Montana, April 20, 2006.-Continued

[Sites are listed in downstream order. Shaded portion highlights the time difference in readings. Abbreviation: $\mathrm{ft}^{3} / \mathrm{s}$, cubic feet per second; $\mu \mathrm{S} / \mathrm{cm}, \mathrm{microsiemens}$ per centimeter at 25 degrees Celsius; SAR, sodium-adsorption ratio. Symbol: --, no data]

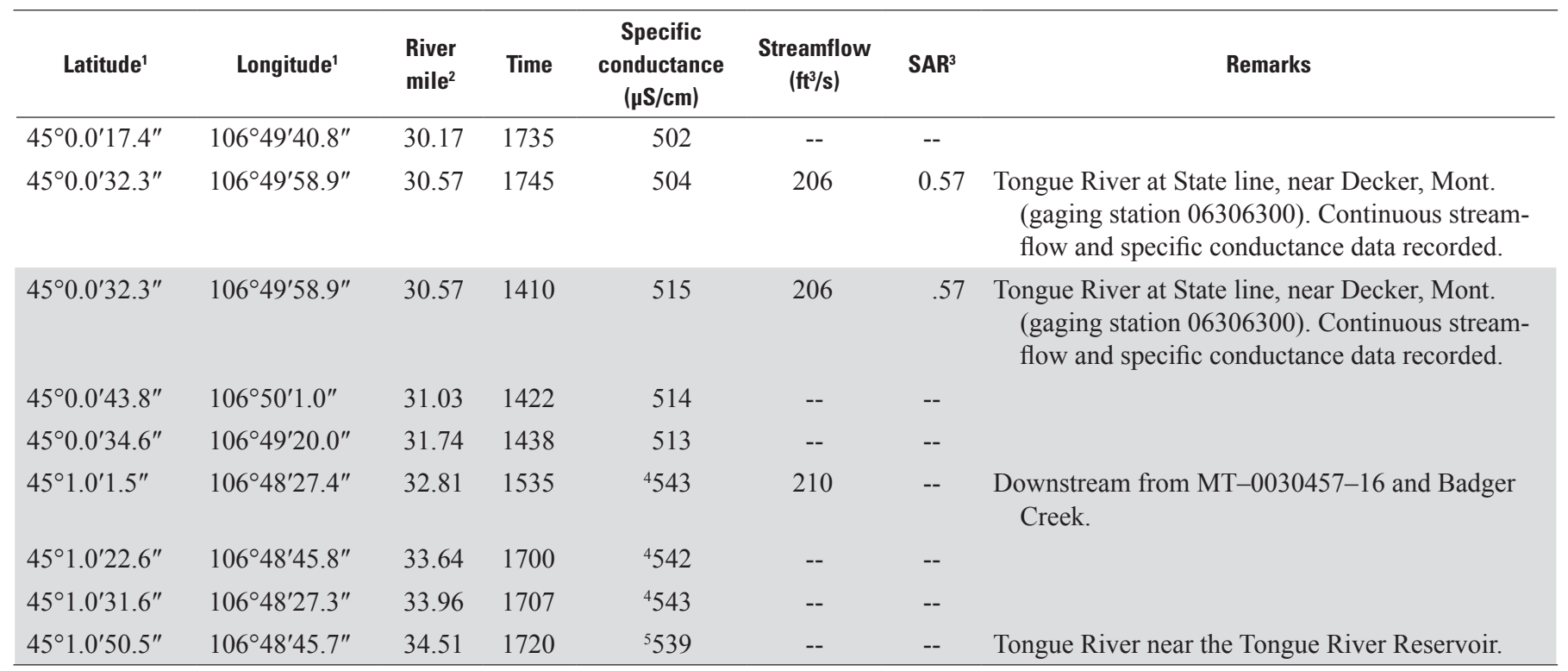

${ }^{1}$ Latitude and longitude, in degrees, minutes, and seconds, are referenced to the North American Datum of 1927 (NAD 27).

${ }^{2}$ Distance downstream from Tongue River at Monarch (station 06299980).

${ }^{3}$ Estimated from real-time specific conductance by using linear-regression equations (Cannon and others, 2007).

${ }^{4}$ From cross-sectional average.

${ }^{5}$ Probably affected by backwater from reservoir.

\section{Streamflow}

Streamflow was measured at most CBM discharge outfalls volumetrically by using a calibrated collection container and stopwatch (Rantz and others, 1982). Three measurements were made and the results were averaged. CBM discharge at outfall MT-0030457-15 was too large to measure volumetrically; therefore, the flow was estimated on the basis of visual estimates of velocity and cross-sectional area (table 2). CBM discharge at outfall MT-0030457-16 was estimated using a mass-balance calculation (table 4) because the flow could not be directly measured or estimated visually (see the "MassBalance and Other Calculations" section).

Streamflow for the Tongue River (Tongue River at Monarch and Tongue River at State line) and the two major tributaries (Goose Creek near Acme and Prairie Dog Creek near Acme) was obtained from stage-discharge rating tables (Rantz and others, 1982). For tributaries without gaging stations, streamflow was determined either by using the floating-object method or by using a current meter (Rantz and others, 1982), depending on the amount of flow.

\section{Water Quality}

Specific conductance of the Tongue River was measured at multiple locations throughout the study reach and at points upstream and downstream from inflows (figs. 1 and 2). Downstream from inflows, the degree of mixing was determined by measuring $\mathrm{SC}$ along a transect across the river. If the river appeared to be well mixed on the basis of the cross-sectional data, the SC at midstream was recorded. If mixing was not complete, the average value for the cross-sectional data was recorded. SC also was measured at most inflow sites. In addition, continuous SC was monitored before, during, and after each synoptic sampling trip at four gaging stations (Tongue River at Monarch, Goose Creek near Acme, Prairie Dog Creek near Acme, and Tongue River at State line) and made available to the public in real-time on the World Wide Web (http:// waterdata.usgs.gov).

Specific-conductance measurements made with the same meter on the same day are assumed to have an associated error of no more than plus or minus 1 percent of the measured value, which is the accuracy listed in the manufacturer's 
Table 4. Physical and chemical characteristics of inflows to the upper Tongue River, Wyoming and Montana, April 20, 2006.

[Site names for permitted coal-bed methane discharges correspond to names designated by the Montana Department of Environmental Quality (for example, MT-0030457-009). Sites are listed in downstream order. Sodium-adsoprtion ratio was calculated from laboratory analytical data unless otherwise noted. Site type: C, permitted coal-bed methane discharge; $\mathrm{T}$, tributary. Abbreviations: $\mathrm{ft}^{3} / \mathrm{s}$, cubic feet per second; $\mu \mathrm{S} / \mathrm{cm}$, microsiemens per centimeter at 25 degrees Celsius; mg/L, milligrams per liter; SAR, sodium-adsorption ratio. Symbols: <, less than; --, no data]

\begin{tabular}{|c|c|c|c|c|c|c|c|c|c|c|c|c|}
\hline Site name & $\begin{array}{l}\text { U.S. Geological } \\
\text { Survey gaging- } \\
\text { station or } \\
\text { miscellaneous } \\
\text { measurement } \\
\text { number }\end{array}$ & Latitude $^{1}$ & Longitude $^{1}$ & $\begin{array}{l}\text { River } \\
\text { mile }^{2}\end{array}$ & Time & $\begin{array}{l}\text { Site } \\
\text { type }\end{array}$ & $\begin{array}{c}\text { Stream- } \\
\text { flow } \\
\left(\mathrm{ft}^{3} / \mathrm{s}\right)\end{array}$ & $\begin{array}{c}\text { Specific } \\
\text { conductance } \\
(\mu \mathrm{S} / \mathrm{cm})\end{array}$ & $\begin{array}{l}\text { Dissolved } \\
\text { sodium } \\
\text { (mg/L) }\end{array}$ & $\begin{array}{l}\text { Dissolved } \\
\text { calcium } \\
\text { (mg/L) }\end{array}$ & $\begin{array}{c}\text { Dissolved } \\
\text { magnesium } \\
\text { (mg/L) }\end{array}$ & SAR \\
\hline $\begin{array}{l}\text { Goose Creek near Acme, } \\
\text { Wyo. }\end{array}$ & 06305700 & $44^{\circ} 54^{\prime} 44.6^{\prime \prime}$ & $106^{\circ} 58^{\prime} 45.3^{\prime \prime}$ & 2.81 & 850 & $\mathrm{~T}$ & 73.0 & 562 & - & -- & -- & ${ }^{3} 0.57$ \\
\hline Youngs Creek at mouth & 445817106544601 & $44^{\circ} 58^{\prime} 17.0^{\prime \prime}$ & $106^{\circ} 54^{\prime} 46.2^{\prime \prime}$ & 16.12 & 1040 & $\mathrm{~T}$ & .96 & 2,020 & 131 & 120 & 173 & 1.8 \\
\hline MT-0030457-009 & -- & $44^{\circ} 59^{\prime} 48.9^{\prime \prime}$ & $106^{\circ} 52^{\prime} 48.4^{\prime \prime}$ & 22.17 & 1310 & $\mathrm{C}$ & Dry & -- & -- & -- & -- & -- \\
\hline MT-0030457-008 & 445955106524801 & $44^{\circ} 59^{\prime} 55.2^{\prime \prime}$ & $106^{\circ} 52^{\prime} 48.0^{\prime \prime}$ & 22.27 & 1330 & $\mathrm{C}$ & .08 & 1,900 & 466 & 5.08 & 1.71 & 46 \\
\hline MT-0030457-002 & -- & $44^{\circ} 59^{\prime} 56.5^{\prime \prime}$ & $106^{\circ} 52^{\prime} 31.9^{\prime \prime}$ & 22.47 & 1350 & $\mathrm{C}$ & Dry & -- & -- & -- & -- & -- \\
\hline $\begin{array}{l}\text { MT-0030457-004, } \\
\text { Diffuser }\end{array}$ & -- & $45^{\circ} 00^{\prime} 10.9^{\prime \prime}$ & $106^{\circ} 52^{\prime} 25.2^{\prime \prime}$ & 22.77 & 1400 & $\mathrm{C}$ & -- & -- & -- & -- & -- & -- \\
\hline MT-0030457-001 & -- & $45^{\circ} 00^{\prime} 17.3^{\prime \prime}$ & $106^{\circ} 52^{\prime} 17.9^{\prime \prime}$ & 23.37 & 1420 & $\mathrm{C}$ & .05 & 1,920 & -- & -- & -- & -- \\
\hline MT-0030457-010 & -- & $45^{\circ} 00^{\prime} 02.7^{\prime \prime}$ & $106^{\circ} 52^{\prime} 11.2^{\prime \prime}$ & 23.67 & 1430 & $\mathrm{C}$ & .02 & 2,000 & -- & -- & -- & -- \\
\hline MT-0030457-005 & 445955106515801 & $44^{\circ} 59^{\prime} 54.8^{\prime \prime}$ & $106^{\circ} 51^{\prime} 57.6^{\prime \prime}$ & 23.87 & 1445 & $\mathrm{C}$ & .04 & 1,720 & 404 & 3.82 & 1.27 & 46 \\
\hline $\begin{array}{l}\text { MT-0030457-006, } \\
\text { Diffuser }\end{array}$ & -- & $45^{\circ} 00^{\prime} 09.7^{\prime \prime}$ & $106^{\circ} 51^{\prime} 16.2^{\prime \prime}$ & 24.97 & 1510 & $\mathrm{C}$ & -- & -- & -- & -- & -- & -- \\
\hline MT-0030457-007 & -- & $45^{\circ} 00^{\prime} 15.8^{\prime \prime}$ & $106^{\circ} 51^{\prime} 10.8^{\prime \prime}$ & 25.07 & 1520 & $\mathrm{C}$ & .08 & 2,070 & -- & -- & -- & -- \\
\hline Squirrel Creek at mouth & -- & $45^{\circ} 00^{\prime} 15.5^{\prime \prime}$ & $106^{\circ} 51^{\prime} 04.2^{\prime \prime}$ & 25.12 & 1415 & $\mathrm{~T}$ & -- & -- & -- & -- & -- & -- \\
\hline MT-0030457-011 & -- & $44^{\circ} 59^{\prime} 55.1^{\prime \prime}$ & $106^{\circ} 50^{\prime} 52.0^{\prime \prime}$ & 25.47 & 1535 & $\mathrm{C}$ & Dry & -- & -- & -- & -- & -- \\
\hline MT-003457-014 & -- & $44^{\circ} 59^{\prime} 53.5^{\prime \prime}$ & $106^{\circ} 50^{\prime} 53.3^{\prime \prime}$ & 25.62 & 1535 & $\mathrm{C}$ & .05 & 1,820 & -- & -- & -- & -- \\
\hline $\begin{array}{l}\text { Prairie Dog Creek near } \\
\text { Acme, Wyo. }\end{array}$ & 06306250 & $44^{\circ} 59^{\prime} 19.6^{\prime \prime}$ & $106^{\circ} 50^{\prime} 47.9^{\prime \prime}$ & 27.99 & 1620 & $\mathrm{~T}$ & 11.0 & 1,400 & -- & -- & -- & ${ }^{3} 1.33$ \\
\hline MT-0030457-012 & -- & $44^{\circ} 59^{\prime} 49.6^{\prime \prime}$ & $106^{\circ} 49^{\prime} 26.0^{\prime \prime}$ & 28.71 & 1655 & $\mathrm{C}$ & .03 & 1,970 & -- & -- & -- & -- \\
\hline MT-0030457-013 & 450007106495201 & $45^{\circ} 00^{\prime} 07.2^{\prime \prime}$ & $106^{\circ} 49^{\prime} 51.7^{\prime \prime}$ & 29.91 & 1725 & $\mathrm{C}$ & .05 & 1,920 & 471 & 3.26 & .99 & 59 \\
\hline MT-0030457-015 & -- & $45^{\circ} 00^{\prime} 17.0^{\prime \prime}$ & $106^{\circ} 49^{\prime \prime} 40.0^{\prime \prime}$ & 30.17 & 1735 & $\mathrm{C}$ & Dry & -- & -- & -- & -- & -- \\
\hline $\begin{array}{l}\text { MT-0030457-016, } \\
\text { Diffuser }\end{array}$ & -- & $45^{\circ} 00^{\prime} 46.4^{\prime \prime}$ & $106^{\circ} 49^{\prime} 05.8^{\prime \prime}$ & 31.97 & 1445 & $\mathrm{C}$ & ${ }^{5} 4.25$ & ${ }^{6} 1,900$ & -- & -- & -- & -- \\
\hline Badger Creek at mouth & -- & $45^{\circ} 00^{\prime} 42.9^{\prime \prime}$ & $106^{\circ} 48^{\prime} 44.5^{\prime \prime}$ & 32.30 & 1515 & $\mathrm{~T}$ & ${ }^{7} 0$ & 8,520 & -- & -- & -- & -- \\
\hline
\end{tabular}

${ }^{1}$ Latitude and longitude, in degrees, minutes and seconds, are referenced to the North American Datum of 1927 (NAD 27).

${ }^{2}$ Distance downstream from Tongue River at Monarch (station 06299980).

${ }^{3}$ Estimated from real-time specific conductance by using linear-regression equations (Cannon and others, 2007).

${ }^{4}$ Outfall for discharge relocated to MT-0030457-016.

${ }^{5}$ Estimated by using a mass-balance calculation.

${ }^{6}$ Flow-weighted average of specific-conductance values for the other outfalls.

${ }^{7}$ Pooled water, no flow. 
specifications for the instrument used to measure SC (Orion Research, Inc., 1991; YSI, Incorporated, 2004). Comparison of data collected with different meters on the same day (as during the April 2006 synoptic sampling trip) or the same meter on different days (as during the September 2005 synoptic sampling trip) indicated that measurements could have an associated error of about 2-3 percent owing to variations introduced by recalibrating the instrument each day or variations between meters.

Water-quality samples were collected from selected inflows (tables 2 and 4). These samples were collected at a single point in the center of flow (tributaries) or directly from the CBM discharge outfalls. Samples were passed through a syringe filter ( 0.45 -micrometer pore size), preserved, and then analyzed at the USGS National Water Quality Laboratory in Denver, Colo., for dissolved calcium, magnesium, and sodium (Fishman, 1993).

Quality-assurance procedures used for the collection and field processing of water-quality data and samples are described by Ward and Harr (1990), Lambing (2006), Horowitz and others (1994), Edwards and Glysson (1999), and U.S. Geological Survey (variously dated). Standard procedures used by the National Water Quality Laboratory for internal sample handling and quality assurance are described by Friedman and Erdmann (1982), Jones (1987), and Pritt and Raese (1995).

The quality of analytical results reported for water-quality samples was not evaluated directly for this study but likely is similar to the quality reported by Cannon and others (2007) for samples collected along the Tongue River during water years 2004-06. Cannon and others (2007) evaluated the quality of analytical results through the use of quality-control samples that were analyzed concurrently in the laboratory with the environmental samples. These quality-control samples consisted of field replicates and blanks, which provided quantitative information on the precision and bias of the overall field and laboratory process. The average relative percent difference (RPD) for calcium, magnesium, and sodium concentrations in the 14 field-replicate samples was 1.3 percent, 0.8 percent, and 1.3 percent, respectively. The maximum RPD for these constituents was 3.1 percent, 2.4 percent, and 4.0 percent, respectively. RPD values were calculated by dividing the difference in concentration of the two replicates by the average of the two concentrations, and then multiplying the result by 100. Concentrations of calcium, magnesium, and sodium were less than the laboratory reporting level in the 12 field-blank samples collected during the same period.

\section{Mass-Balance and Other Calculations}

Mass-balance calculations were used to estimate streamflow, SC, SAR, or ion concentrations for some sites along the Tongue River where one (or more) of these physical or chemical characteristics was not measured. Mass-balance calculations also were used to project potential increases in SC and SAR resulting from direct CBM discharge to the Tongue
River. These calculations are based on the principle of conservation of mass, as expressed in the following two equations:

$$
C_{\text {down }} Q_{\text {down }}=\left(C_{\text {up }} Q_{\text {up }}\right)+\left(C_{\text {inf }} Q_{\text {inf }}\right),
$$

and

$$
Q_{\text {down }}=Q_{u p}+Q_{\text {inf }},
$$

where

$C_{\text {down }}$ is concentration, in milligrams per liter, specific conductance, in microsiemens per centimeter at 25 degrees Celsius, or SAR of the Tongue River downstream from an inflow;

$Q_{\text {down }} \quad$ is streamflow of the Tongue River downstream from an inflow, in cubic feet per second;

$C_{u p} \quad$ is concentration, in milligrams per liter, specific conductance, in microsiemens per centimeter at 25 degrees Celsius, or SAR of the Tongue River upstream from an inflow;

$Q_{u p} \quad$ is streamflow of the Tongue River upstream from an inflow, in cubic feet per second;

$C_{i n f}$ is concentration, in milligrams per liter, specific conductance, in microsiemens per centimeter at 25 degrees Celsius, or SAR of the inflow; and

$Q_{i n f} \quad$ is streamflow of the inflow, in cubic feet per second.

Implicit in the mass-balance calculations used for estimating $\mathrm{SC}$ is the assumption that $\mathrm{SC}$ is a chemically conservative characteristic that can be used as a proxy for dissolved-solids concentration. Similarly, implicit in the mass-balance calculations used for estimating SAR is the assumption that SAR is a chemically conservative characteristic. However, this assumption for SAR is not always valid, particularly if the major-ion composition of the stream and inflow is not similar.

To evaluate potential effects of CBM discharge on the Tongue River, historical information on the SC and SAR in the river prior to $\mathrm{CBM}$ development was needed for a range of streamflow conditions. This information was estimated for Tongue River at State line using linear regression (Helsel and Hirsch, 2002) and historical streamflow and water-quality data. One regression equation was developed for estimating historical SC from streamflow. Another set of regression equations was developed for estimating historical concentrations of calcium, magnesium, and sodium from streamflow. The uncertainty associated with regression estimates may be represented by the 95 -percent prediction interval, which represents the range of values within which the true value will occur 95 percent of the time (Helsel and Hirsch, 2002). 
The average value of the SC or concentration of a major ion in the combined flow from multiple sources (for example, several CBM discharges) was needed to assist in interpreting water-quality data. These values were determined by using the flow-weighted average. The flow-weighted average was calculated by first summing the products of the SC or ion concentration and the flow for each source and then dividing the resulting sum by the sum of the flows for all sources.

\section{Specific Conductance along the Upper Tongue River}

Field conditions were appropriate for conducting synoptic sampling during both sampling periods. During both synoptic sampling trips, the weather was sunny and warm, and no precipitation fell in the area. Streamflows during the September 2005 sampling trip generally were steady (fig. 3), varying by about 10 percent or less during the sampling period. SC varied between 625 and $645 \mu \mathrm{S} / \mathrm{cm}$ at Tongue River at State line (about a 3-percent change; fig. 4). Three days prior to the sampling, 0.23 inch (in.) of rain fell at the precipitation station in Sheridan, Wyo. (National Oceanic and Atmospheric Administration, 2007). This rain caused increased streamflow at Tongue River at Monarch and at Goose Creek near Acme prior to the September 2005 sampling trip, but streamflows in the main stem decreased and remained fairly constant at these sites during the sampling period (fig. 3). At Tongue River at State line, streamflow increased gradually during the sampling trip (about an 11-percent increase), most likely representing arrival of the increased streamflow recorded at Tongue River at Monarch and at Goose Creek near Acme during the preceding days. This increasing streamflow could have had a small effect on the results of the sampling. On the basis of field observations, some water was being diverted for irrigation during the September 2005 sampling. Although the amount of water being diverted was not determined, the small amount of water probably had little effect on the sampling results.

Streamflows during the April 2006 synoptic sampling trip were higher and more varied than during the September 2005 sampling trip, primarily owing to diurnal flow patterns at several sites (fig. 5). Streamflows during the April 2006 sampling period varied by about 20 percent or less at the Tongue River at State line and Goose Creek near Acme gaging stations. Streamflows at Tongue River at Monarch varied about 50 percent while streamflows at Prairie Dog Creek near Acme decreased sharply (about 60 percent) during the sampling period. SC varied between 492 and $495 \mu \mathrm{S} / \mathrm{cm}$ at Tongue River at State line (about a 1-percent change; fig. 6). Three days prior to the sampling, 0.24 in. of rain fell at the precipitation station in Sheridan, Wyo. (National Oceanic and Atmospheric Administration, 2007). Streamflow increased slightly to moderately on April 17 and 18 at all sites (fig. 5). On the basis of field observations, water was not being diverted for irrigation during the April 2006 sampling trip.

\section{Specific Conductance, September 2005}

Specific conductance of the upper Tongue River generally increased in the downstream direction during the synoptic investigation on September 27-28, 2005 (fig. 7 and table 1). The overall increase in SC from $420 \mu \mathrm{S} / \mathrm{cm}$ at Tongue River at Monarch (river mile 0.00 ) to $625 \mu \mathrm{S} / \mathrm{cm}$ at Tongue River at State line (river mile 30.57) was 49 percent. The increases in SC downstream from Goose Creek and Prairie Dog Creek were large. SC increased 24 percent from $420 \mu \mathrm{S} / \mathrm{cm}$ upstream from Goose Creek to $520 \mu \mathrm{S} / \mathrm{cm}$ downstream from Goose Creek (table 1). SC increased 15 percent from $544 \mu \mathrm{S} / \mathrm{cm}$ upstream from Prairie Dog Creek to $625 \mu \mathrm{S} / \mathrm{cm}$ downstream from Prairie Dog Creek.

Specific conductance of the Tongue River remained essentially constant between Tongue River at Monarch and Goose Creek and then between Goose Creek and Prairie Dog Creek. There was an increase in SC of about 3 percent near Ash Creek where the September 27 measurement reach began and the September 28 reach ended (fig. 7 and table 1). This change in SC likely is a combined result of differences in SC in the river and in calibration of the $\mathrm{SC}$ meter on the two sampling days. Although the SC of Ash and Youngs Creeks was high $(1,700$ and $1,260 \mu \mathrm{S} / \mathrm{cm}$, respectively), these tributaries had a negligible effect on the SC of the Tongue River because of their very small flow (table 2). Also, the irrigation return flow (river mile 20.60) had negligible effect because the SC of the return flow was similar to that of the Tongue River (530 and $541 \mu \mathrm{S} / \mathrm{cm}$, respectively; tables 1 and 2).

The only other visible inflows to the Tongue River were the $15 \mathrm{CBM}$ discharge outfalls. Two of these outfalls were dry, and one had too little streamflow to allow measurement of SC. SC of the 12 remaining CBM discharges ranged from 1,750 to $2,440 \mu \mathrm{S} / \mathrm{cm}$ (table 2), and the flow-weighted average was $2,260 \mu \mathrm{S} / \mathrm{cm}$. The combined streamflow from all measured CBM discharge outfalls was $3.12 \mathrm{ft}^{3} / \mathrm{s}$ or $1,400 \mathrm{gal} / \mathrm{min}$. About two-thirds of this flow was discharged from outfall MT-0030457-015 (table 2), which was about 2 river miles downstream from Prairie Dog Creek.

CBM discharge from the outfalls upstream from Prairie Dog Creek had no measurable effect on SC in the Tongue River (fig. 7). The effect of CBM discharge on the Tongue River downstream from Prairie Dog Creek was not measured directly because inflows from CBM discharges and Prairie Dog Creek did not completely mix in the main stem before the river reached the next downstream CBM discharge. However, between the Tongue River immediately upstream from Prairie Dog Creek and Tongue River at State line, SC increased $81 \mu \mathrm{S} / \mathrm{cm}$, from 544 to $625 \mu \mathrm{S} / \mathrm{cm}$ (about 15 percent; table 1 and fig. 7). The contribution of CBM discharge to the increase in $\mathrm{SC}$ in this reach was estimated by using equations 1 and 2, the SC and streamflow data for Tongue River at State line $\left(625 \mu \mathrm{S} / \mathrm{cm}, 195 \mathrm{ft}^{3} / \mathrm{s}\right)$, the flow-weighted average SC of the 12 measured CBM discharges $(2,260 \mu \mathrm{S} / \mathrm{cm})$, and the sum of the streamflow for the three CBM discharges downstream from Prairie Dog Creek $\left(2.09 \mathrm{ft}^{3} / \mathrm{s}\right)$. The estimated increase in 

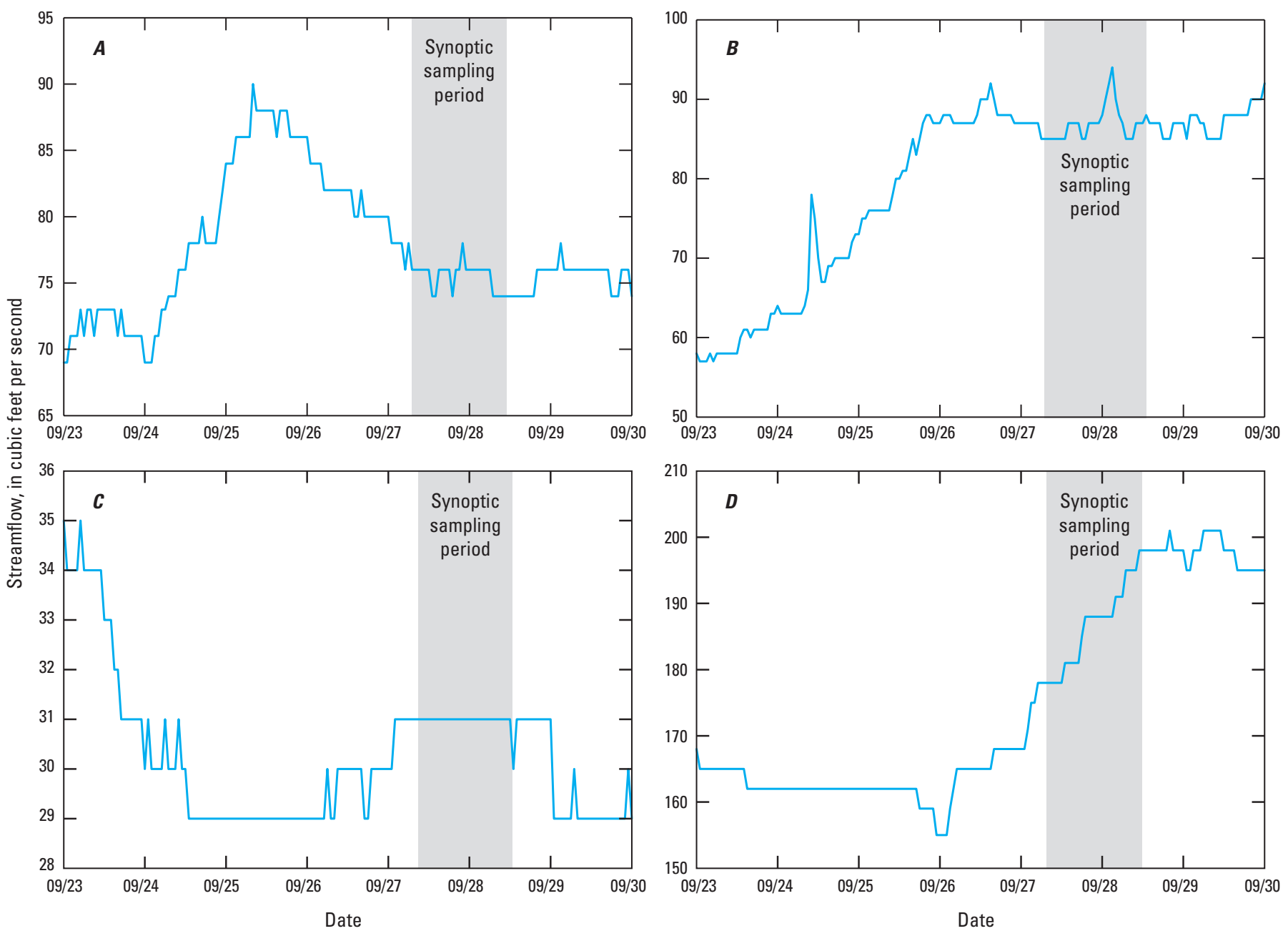

Figure 3. Continuous streamflow during September 23-30, 2005. $A$, Tongue River at Monarch, Wyo. (06299980). B, Goose Creek near Acme, Wyo. (06305700). C, Prairie Dog Creek near Acme, Wyo. (06306250). D, Tongue River at State line, near Decker, Mont. (06306300).

$\mathrm{SC}$ at Tongue River at State line resulting from the three CBM discharges downstream from Prairie Dog Creek combined was $18 \mu \mathrm{S} / \mathrm{cm}$ (a 2.9-percent increase). The remaining increase of $63 \mu \mathrm{S} / \mathrm{cm}$ in this reach was attributed to Prairie Dog Creek.

The increase in SC in the Tongue River resulting from the inflow of Prairie Dog Creek could have been calculated by using equations 1 and 2 and data from the Prairie Dog Creek near Acme gaging station. However, because of the difference between $\mathrm{SC}$ values from the continuous monitor at the station on Prairie Dog Creek near Acme (about 1 mile upstream from the mouth; figs. 1 and 4) and the measured value at the mouth during the synoptic investigation (table 2), the calculation method of subtracting out the outfalls (described in previous paragraph) is better constrained because the synoptically measured values are spatially and temporally more representative and consistent than the values from the SC monitor at Prairie Dog Creek near Acme.

The estimated increase in SC at Tongue River at State line resulting from all $15 \mathrm{CBM}$ discharge outfalls was
$27 \mu \mathrm{S} / \mathrm{cm}$ (a 4.5-percent increase). This increase in SC was determined by using equations 1 and 2, SC and streamflow data for Tongue River at State line $\left(625 \mu \mathrm{S} / \mathrm{cm}, 195 \mathrm{ft}^{3} / \mathrm{s}\right)$, the flow-weighted average SC of the 12 measured CBM discharge outfalls $(2,260 \mu \mathrm{S} / \mathrm{cm})$, and the combined streamflow of all measured outfalls $\left(3.12 \mathrm{ft}^{3} / \mathrm{s}\right)$. On the basis of relative flow rates among the outfalls, the large flow (estimated $2.0 \mathrm{ft}^{3} / \mathrm{s}$ ) from CBM discharge outfall MT-0030457-15 contributed about 63 percent, or about $17 \mu \mathrm{S} / \mathrm{cm}$, of the $27-\mu \mathrm{S} / \mathrm{cm}$ increase.

\section{Specific Conductance, April 2006}

The SC of the Tongue River generally increased in the downstream direction and increased substantially downstream from Goose and Prairie Dog Creeks during the synoptic investigation on April 20, 2006 (fig. 8 and table 3). This downstream trend in $\mathrm{SC}$ was similar to the trend in September 

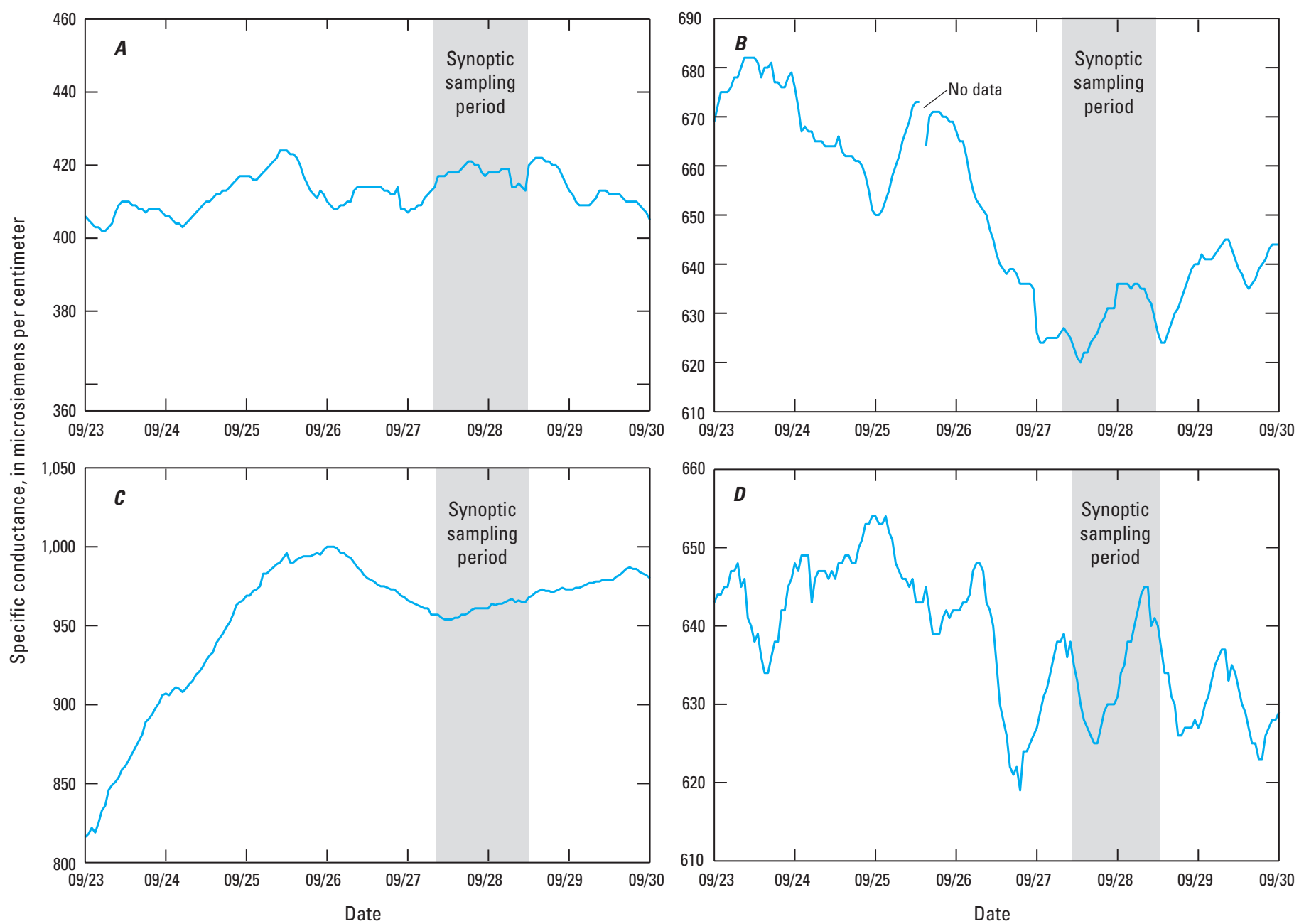

Figure 4. Continuous specific conductance during September 23-30, 2005. A, Tongue River at Monarch, Wyo. (06299980). B, Goose Creek near Acme, Wyo. (06305700). C, Prairie Dog Creek near Acme, Wyo. (06306250). D, Tongue River at State line, near Decker, Mont. (06306300).

2005. The overall increase from $373 \mu \mathrm{S} / \mathrm{cm}$ at river mile 0.00 to $543 \mu \mathrm{S} / \mathrm{cm}$ at river mile 33.96 in April 2006 was 46 percent. $\mathrm{SC}$ in the Tongue River increased from $378 \mu \mathrm{S} / \mathrm{cm}$ upstream from Goose Creek to $439 \mu \mathrm{S} / \mathrm{cm}$ downstream from Goose Creek (a 16-percent increase). SC increased from $439 \mu \mathrm{S} / \mathrm{cm}$ upstream from Prairie Dog Creek to $492 \mu \mathrm{S} / \mathrm{cm}$ downstream from Prairie Dog Creek (a 12-percent increase). These increases generally were similar to the increases (24 percent at Goose Creek and 15 percent at Prairie Dog Creek) in September 2005. Locations of measurement sites referred to as "upstream" or "downstream" from river inflows varied slightly between the two synoptic sampling trips.

Specific conductance in the reach between Goose Creek and the Montana-Wyoming State line was more varied in April 2006 than in September 2005 (figs. 7 and 8). In the upper part of this reach in April 2006, SC increased about 7 percent from $439 \mu \mathrm{S} / \mathrm{cm}$ at river mile 3.51 to $469 \mu \mathrm{S} / \mathrm{cm}$ at river mile 10.85 and then remained nearly constant to river mile 12.50 (upstream of Ash Creek; fig. 8 and table 3). No visible inflows occurred in this reach. Continuing downstream, SC decreased about 5 percent from $452 \mu \mathrm{S} / \mathrm{cm}$ at river mile 12.50 to $428 \mu \mathrm{S} / \mathrm{cm}$ at the Montana-Wyoming State line (river mile 21.74; fig. 8 and table 3). The decreasing SC in this reach may have masked any increase in $\mathrm{SC}$ in the main stem that might have been contributed by Youngs Creek, which was flowing $0.96 \mathrm{ft}^{3} / \mathrm{s}$ with a SC of $2,020 \mu \mathrm{S} / \mathrm{cm}$ (table 4). The irrigation return flow observed in September 2005 at river mile 20.60 (table 2) was not found during April 2006. The overall downstream pattern of increasing and then decreasing SC between Goose Creek and the Montana-Wyoming State line was not observed during September 2005 and is thought to reflect the varying SC recorded at Tongue River at Monarch during the 24-hour period prior to the start of sampling (fig. 6).

As observed during the September 2005 synoptic sampling trip, the only visible inflows besides tributaries to the Tongue River in April 2006 were the 15 CBM discharge outfalls. Between the September 2005 and April 2006 synoptic sampling trips, three of the outfalls in the study reach 

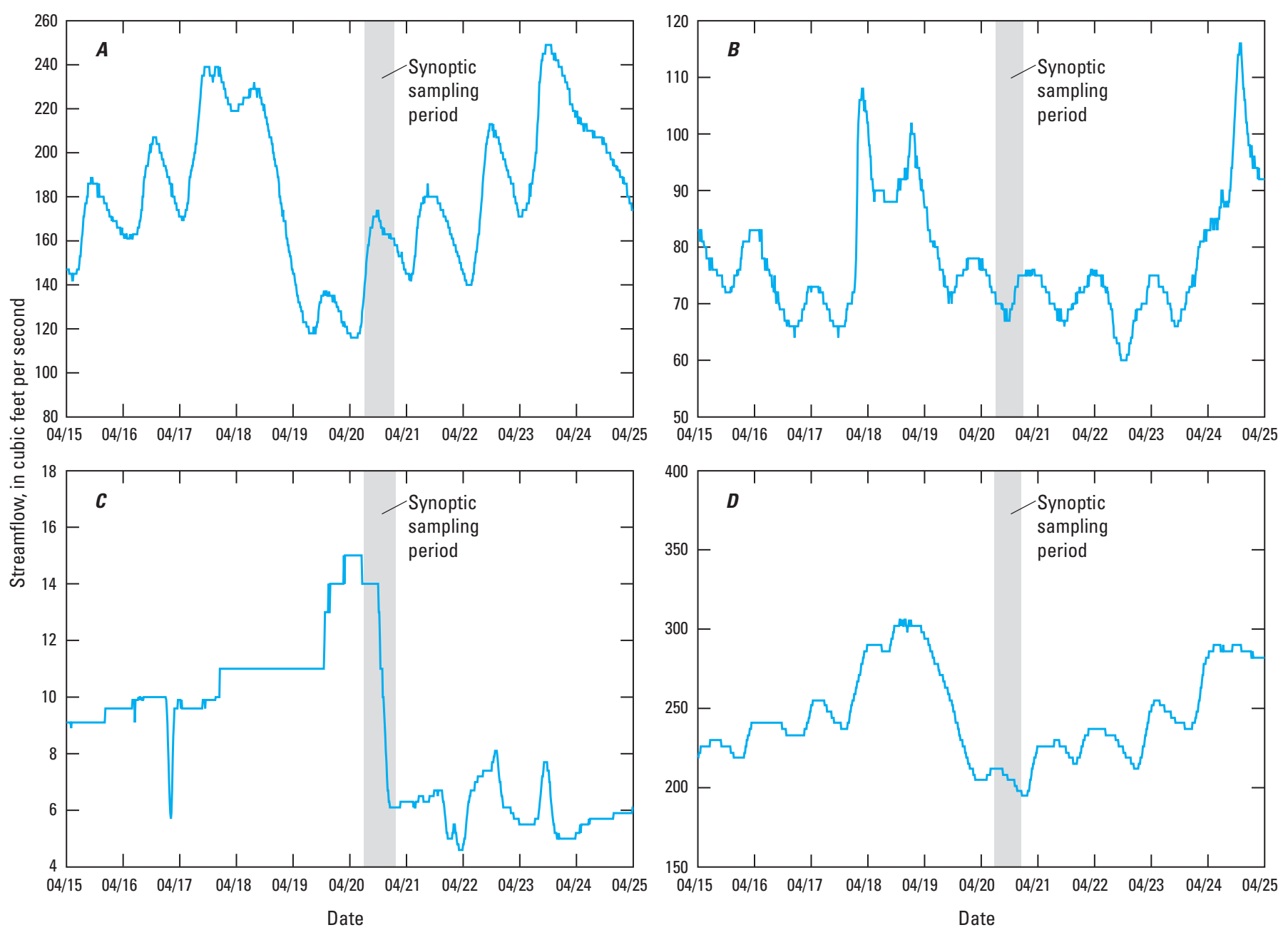

Figure 5. Continuous streamflow during April 15-25, 2006. A, Tongue River at Monarch, Wyo. (06299980). B, Goose Creek near Acme, Wyo. (06305700). C, Prairie Dog Creek near Acme, Wyo. (06306250). D, Tongue River at State line, near Decker, Mont. (06306300).

were equipped with effluent diffusers (table 4). Diffusers were installed across the width of the streambed and generally perpendicular to streamflow to facilitate rapid mixing of CBM discharge with river water. Because the diffusers were submerged, streamflow and SC of CBM discharge could not be measured at these sites. Four CBM discharge outfalls were dry. In April 2006, the SC of the eight measured CBM discharges ranged from 1,720 to $2,070 \mu \mathrm{S} / \mathrm{cm}$ (table 4) and the flow-weighted average was $1,900 \mu \mathrm{S} / \mathrm{cm}$. Streamflow from the eight measured outfalls varied from 0.02 to $0.08 \mathrm{ft}^{3} / \mathrm{s}$ (table 4) and the combined streamflow totaled $0.40 \mathrm{ft}^{3} / \mathrm{s}$. However, this combined flow was only a small percent of the CBM discharge to the Tongue River in the study reach because the flows being discharged from the three diffuser-equipped outfalls were not measured but, based on visual inspection, appeared larger than the flows from any of the measured CBM discharges. The reason that the flow-weighted SC of CBM discharges from April $2006(1,900 \mu \mathrm{S} / \mathrm{cm})$ was lower than the flow-weighted $\mathrm{SC}$ of CBM discharges from September $2005(2,260 \mu \mathrm{S} / \mathrm{cm})$ is not known but may be due to either the absence of SC data for the unmeasured CBM discharge in April 2006 or differences in water-quality characteristics of the coal seams that were being pumped during the two periods.

Specific conductance in the Tongue River increased at three places in the downstream end of the study reach. The first increase of approximately $14 \mu \mathrm{S} / \mathrm{cm}$ (from 428 to $442 \mu \mathrm{S} / \mathrm{cm}$, or 3.3 percent) occurred upstream from Prairie Dog Creek between river miles 21.74 and 25.58 (table 3 and fig. 8). Visible inflows in this reach included Squirrel Creek and $10 \mathrm{CBM}$ discharge outfalls. Squirrel Creek was not measured, and discharge at two of the CBM discharge outfalls was routed through diffusers and could not be measured. Three CBM discharge outfalls were dry. The SC values of the five measured CBM discharge outfalls $(1,720$ to $2,070 \mu \mathrm{S} / \mathrm{cm})$ upstream from Prairie Dog Creek were similar, and these five outfalls had a combined discharge of $0.27 \mathrm{ft}^{3} / \mathrm{s}$. In order to estimate the increase in $\mathrm{SC}$ in the Tongue River resulting from the five measured CBM discharges, equations 1 and 2 were used 

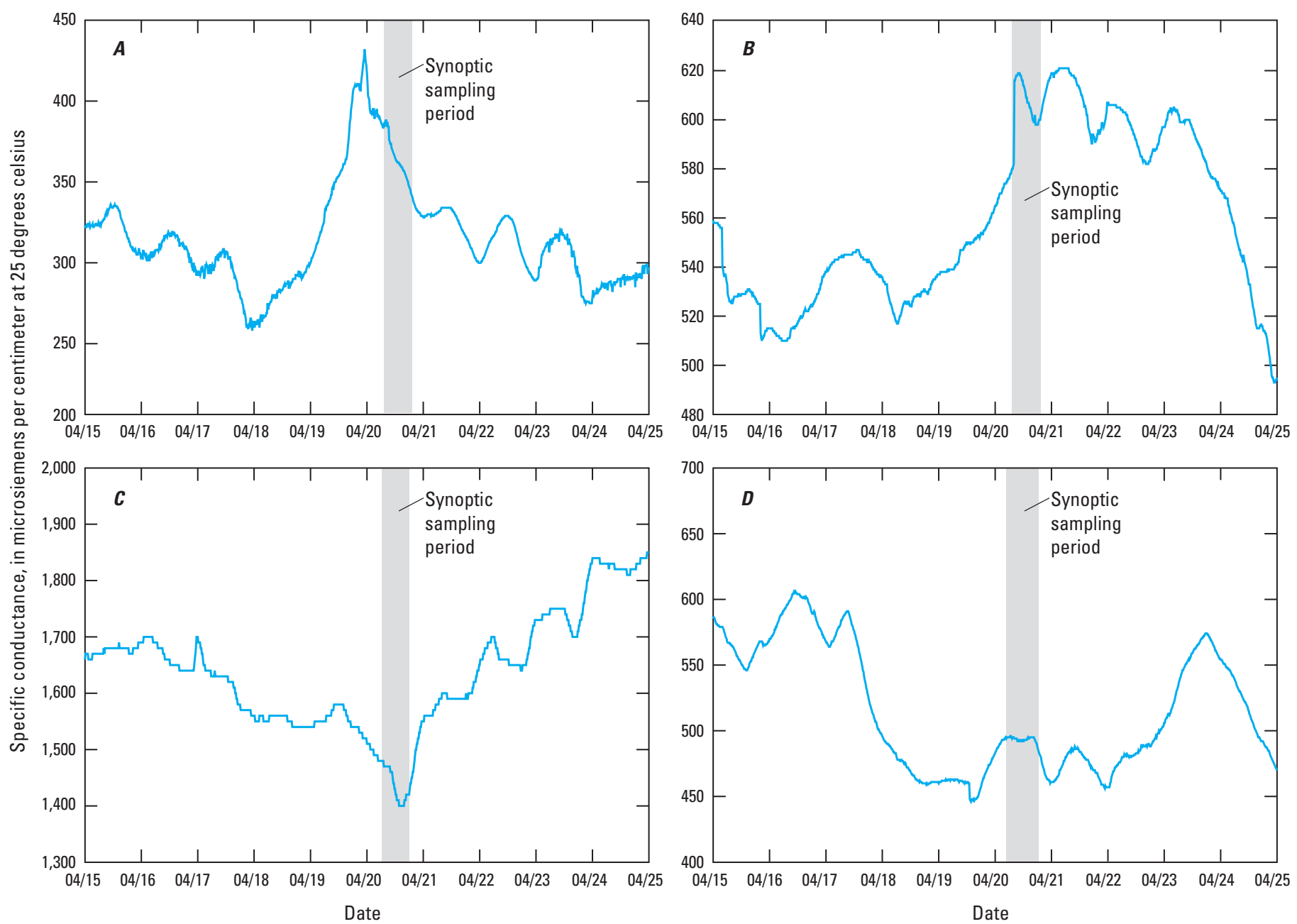

Figure 6. Continuous specific conductance during April 15-25, 2006. A, Tongue River at Monarch, Wyo. (06299980). B, Goose Creek near Acme, Wyo. (06305700). C, Prairie Dog Creek near Acme, Wyo. (06306250). D, Tongue River at State line, near Decker, Mont. (06306300).

along with SC and streamflow data for Tongue River at State line $\left(515 \mu \mathrm{S} / \mathrm{cm}, 206 \mathrm{ft}^{3} / \mathrm{s}\right)$, the flow-weighted average SC $(1,900 \mu \mathrm{S} / \mathrm{cm})$ of all eight measured CBM discharges, and the combined streamflow of the five measured CBM discharges in the reach $\left(0.27 \mathrm{ft}^{3} / \mathrm{s}\right)$. This mass-balance calculation indicated that the five measured CBM discharges upstream from Prairie Dog Creek could increase the SC of the Tongue River only by about $2 \mu \mathrm{S} / \mathrm{cm}$. Thus, other factors, such as diurnal changes in streamflow and SC, Squirrel Creek, groundwater inflow, or the two diffuser-equipped CBM discharge outfalls, account for most of the increase in SC $(12 \mu \mathrm{S} / \mathrm{cm}$, or 86 percent $)$ in this reach of the Tongue River.

Second, specific conductance also increased $12 \mu \mathrm{S} / \mathrm{cm}$ (from 492 to $504 \mu \mathrm{S} / \mathrm{cm}$, or 2.4 percent) in the reach from just downstream from Prairie Dog Creek (river mile 28.82) to Tongue River at State line (river mile 30.57; table 3 and fig. 8). Two CBM discharge outfalls were in this reach (river miles 29.91 and 30.17); the discharge from one was $0.05 \mathrm{ft}^{3} / \mathrm{s}$, and the other was dry. To estimate the increase in SC in the Tongue
River resulting from the flowing CBM discharge, equations 1 and 2 were used along with SC and streamflow data for Tongue River at State line $\left(504 \mu \mathrm{S} / \mathrm{cm}, 206 \mathrm{ft}^{3} / \mathrm{s}\right)$, the flowweighted average SC $(1,900 \mu \mathrm{S} / \mathrm{cm})$ of all eight measured CBM discharges, and the streamflow of the measured CBM discharge $\left(0.05 \mathrm{ft}^{3} / \mathrm{s}\right)$ in the reach. This mass-balance calculation indicated that the one $\mathrm{CBM}$ discharge could increase the $\mathrm{SC}$ of the Tongue River only by an estimated $0.3 \mu \mathrm{S} / \mathrm{cm}$. Therefore, this CBM discharge probably was not the cause of the increase in $\mathrm{SC}$ in this second reach upstream from river mile 30.57. Alternatively, this increase in SC may reflect the influence of groundwater inflow as well as diurnal changes in $\mathrm{SC}$ and streamflow (figs. 5 and 6).

Lastly, the SC increased $30 \mu \mathrm{S} / \mathrm{cm}$ (from 513 to $543 \mu \mathrm{S} / \mathrm{cm}$, or 5.8 percent) between river mile 31.74 and 32.81 (table 3) in the Tongue River downstream from CBM discharge outfall MT-0030457-016 and Badger Creek. Although there was pooled water with an $\mathrm{SC}$ of $8,520 \mu \mathrm{S} / \mathrm{cm}$ at the mouth of Badger Creek (river mile 32.30), no flow was 


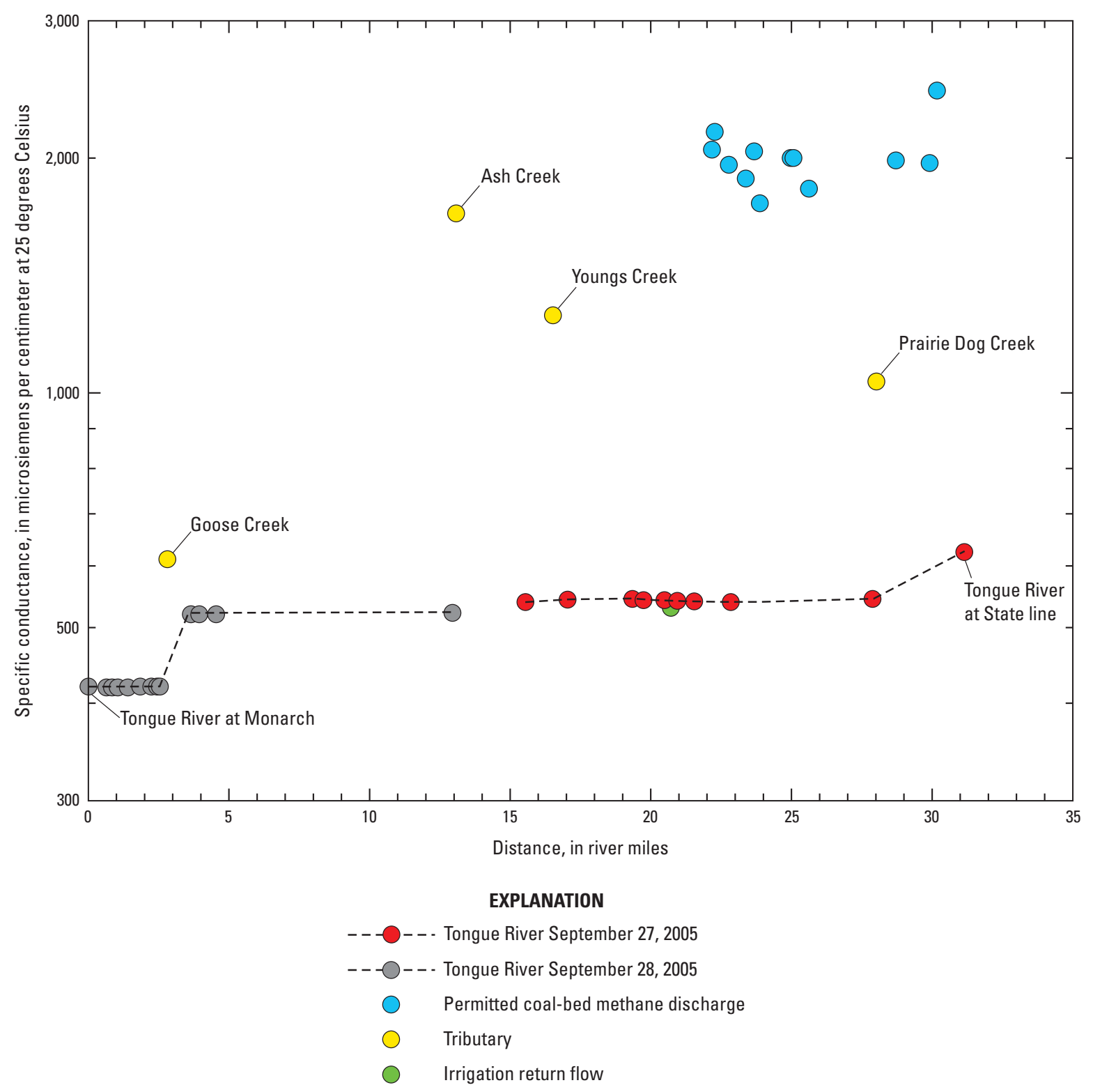

Figure 7. Specific conductance of the upper Tongue River, tributaries, and other inflows between Tongue River at Monarch, Wyo., (06299980), and State line, near Decker, Mont., (06306300), September 27-28, 2005.

observed (table 4); therefore, Badger Creek likely did not cause the increase of SC in the Tongue River. The only other visible inflow to the main stem was CBM discharge outfall MT-0030457-016 (at river mile 31.97). The streamflow and $\mathrm{SC}$ of this discharge could not be measured because the discharge was routed through a diffuser on the streambed. Increased SC values were noted in the main stem of the river directly downstream from the diffuser. The discharge for this outfall was estimated (table 4 ) by using equations 1 and 2. For this estimate, the $\mathrm{SC}$ and streamflow at Tongue River at State line $\left(515 \mu \mathrm{S} / \mathrm{cm}, 206 \mathrm{ft}^{3} / \mathrm{s}\right)$, as well as the $\mathrm{SC}$ of the Tongue River just downstream (at river mile 32.81) from this diffuser $(543 \mu \mathrm{S} / \mathrm{cm})$, were known (table 3$)$. The $\mathrm{SC}$ of the
CBM discharge was assumed to be the flow-weighted average $\mathrm{SC}(1,900 \mu \mathrm{S} / \mathrm{cm})$ of all eight CBM discharges measured on the day of the sampling trip (table 4). This mass-balance calculation indicated that the estimated streamflow for CBM discharge outfall MT-0030457-016 was $4.25 \mathrm{ft}^{3} / \mathrm{s}$ (or $1,910 \mathrm{gal} / \mathrm{min}$ ). This estimate of flow for the outfall is reasonable because it accounts for the measured increase in flow of $4 \mathrm{ft}^{3} / \mathrm{s}$ between Tongue River at State line (206 ft $\mathrm{ft}^{3} / \mathrm{s}$; table 3) and river mile $32.81\left(210 \mathrm{ft}^{3} / \mathrm{s}\right.$; table 3$)$. Thus, the CBM discharge from outfall MT-0030457-16 likely caused the $30-\mu \mathrm{S} / \mathrm{cm}$ increase in $\mathrm{SC}$ in the Tongue River between river miles 31.74 and 32.81 . 


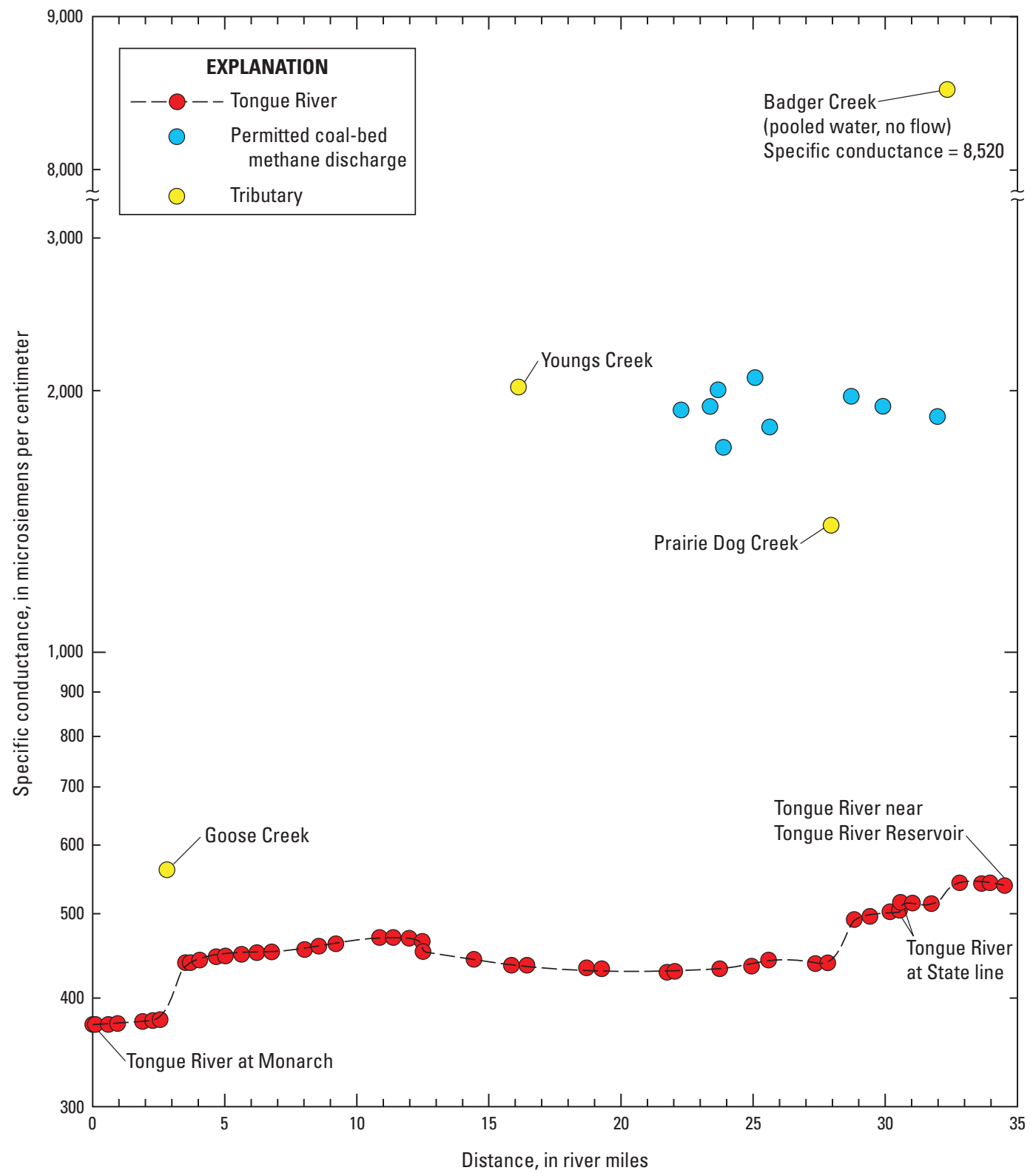

Figure 8. Specific conductance of the upper Tongue River, tributaries, and other inflows between Tongue River at Monarch, Wyo. (06299980), and near the Tongue River Reservoir, Mont., April 20, 2006. 


\section{Potential Water-Quality Effects of Coal-Bed Methane Discharge along the Upper Tongue River}

The dissolved ions that are quantified by SC measurements and that control the SAR of the upper Tongue River come from several sources such as headwater and tributary inflows as well as direct CBM discharge. It is important to understand the relative magnitude of all sources so that the contribution from direct CBM discharge can be put into perspective. To do this, the approximate relative contributions to SC and SAR from tributary and CBM sources were calculated for the April 2006 synoptic sampling trip. These approximate contributions were determined by calculating the load of SC and SAR (streamflow times the value of water-quality characteristic) for each measured inflow to the study reach (tables 3 and 4), summing these contributions (as shown in equation 1), and then determining the percent contribution of each inflow to the total contribution from all inflows. The two largest inflows-Tongue River at Monarch and Goose Creekaccounted for about 80 percent of the SC, whereas direct CBM discharges accounted for only 7 percent (fig. $9 A$ ), which was less than the contribution (12 percent) from Prairie Dog Creek. In contrast, CBM discharges accounted for 68 percent of the SAR (fig. 9B). Note that these calculations are considered approximate because (1) the contributions to SC and SAR from the CBM discharge outfalls that used diffusers (table 4) were not included in the analysis because these discharges were not measured or sampled, and (2) SAR is not as conservative a characteristic of water as is SC.

The data collected during the synoptic sampling trips, as well as the analysis of relative contributions presented in the previous paragraph, are representative only for the day of sampling and the flow conditions encountered at that time. Conditions in the upstream watersheds that affected the export of water and dissolved ions to the Tongue River during the synoptic sampling trips may have been different historically and may be different in the future. For instance, the trend in SC in Goose Creek at a site near Sheridan, Wyo., was small but significantly upward between 1991 and 2005 (Clark and Mason, 2007). The watershed conditions that could affect the export of dissolved ions could include, for instance, climate, land use, irrigation and other agricultural practices, water management, and CBM development. Insufficient hydrologic and water-quality information is available for the inflows to the study reach to assess the potential effects of time-varying watershed conditions.

To broaden the analysis of potential effects to other flow conditions, a series of calculations were made to project the potential increases in $\mathrm{SC}$ and SAR resulting from direct $\mathrm{CBM}$ discharge to the Tongue River. These projections used data for Tongue River at State line and the assumption that all potential CBM discharge occurs upstream from this site. This assumption was true during the September 2005 sampling trip. In April 2006, however, some CBM discharge entered along the 4-mile reach between Tongue River at State line and the reservoir. Therefore, the potential effects projected here are assumed to occur at a downstream site that is referred

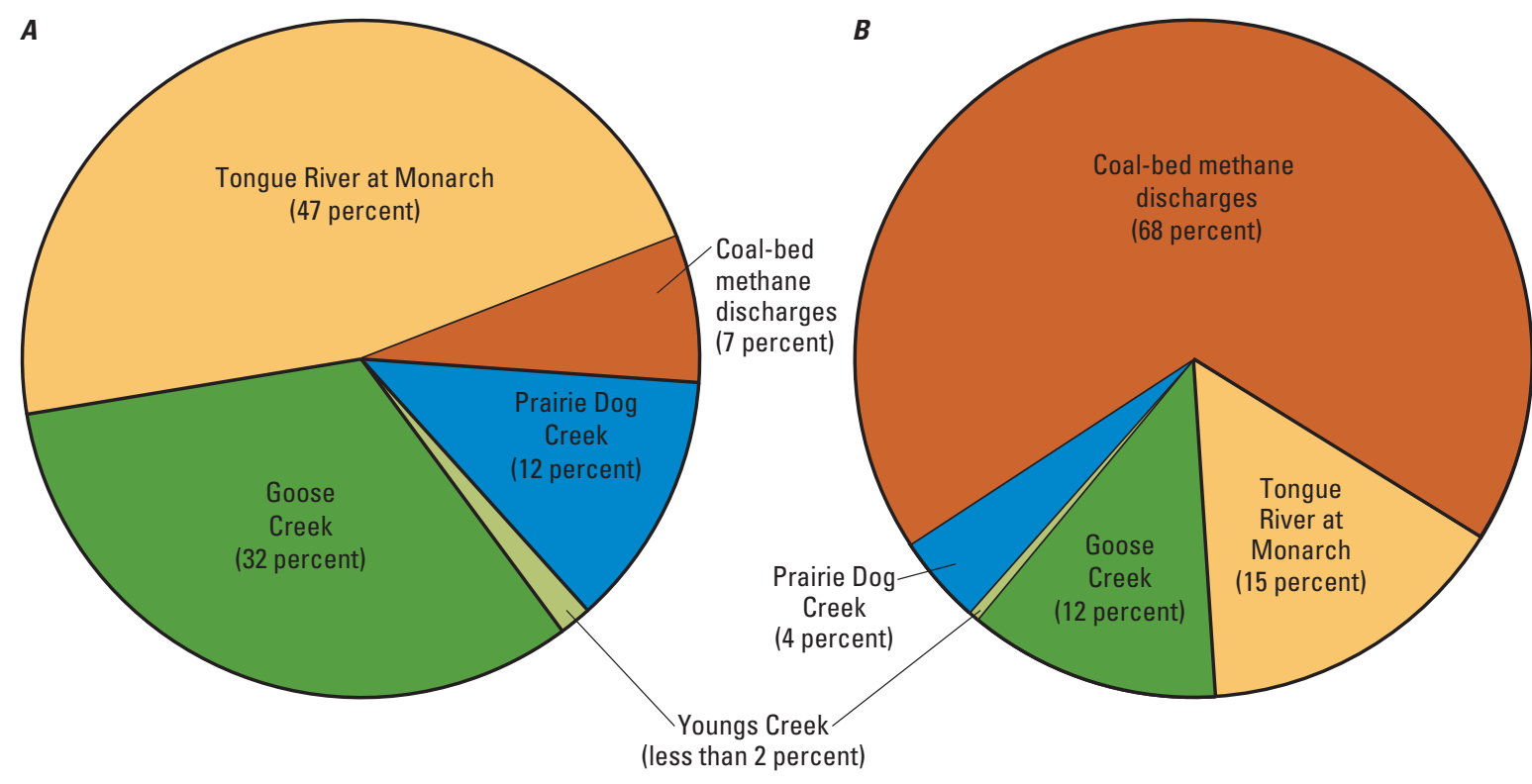

Figure 9. Relative contributions of headwater and tributary inflows to the Tongue River between Monarch, Wyo., and just upstream from the Tongue River Reservoir, Mont., April 20, 2006. A, Specific conductance. B, Sodium-adsorption ratio. 
to as Tongue River near the Tongue River Reservoir (river mile 34.51 ; table 3 and fig. 8). At the time of both synoptic sampling trips, the total combined discharge from all CBM outfalls was not permitted to exceed 2,500 gal $/ \mathrm{min}$, or $5.57 \mathrm{ft}^{3} / \mathrm{s}$ (Montana Department of Environmental Quality, written commun., 2006). Potential effects were calculated for CBM discharge rates equivalent to multiples of this maximum allowable combined discharge.

\section{Specific Conductance}

The potential effects that direct CBM discharge might have on the SC of the Tongue River are dependent on the streamflow in the river and the quantity and chemical characteristics of CBM discharge. Estimates of potential effects of the CBM discharges on the SC of the Tongue River near the Tongue River Reservoir were calculated using a two-step process involving linear regression and mass-balance calculations for a range of streamflow and CBM-discharge conditions. The first step in the calculation process was to derive an equation to estimate the historical SC of the river for a given streamflow in the absence of CBM development. This equation was derived by using linear regression (Helsel and Hirsch, 2002) as well as streamflow and SC data collected at Tongue River at State line during water years 1985-99 (http://waterdata. usgs.gov/mt/nwis), prior to CBM development (fig. 10). Estimated historical SC values were then calculated for a range of streamflows. In the second step, potential increases in the SC of the Tongue River near the Tongue River Reservoir resulting from CBM discharge were projected using mass-balance calculations (equations 1 and 2) for a range of streamflows and $\mathrm{CBM}$ discharges. Data used in the calculations included the estimated historical SC values, a range of streamflows (50 to $3,000 \mathrm{ft}^{3} / \mathrm{s}$ ) representative of most historical flow conditions for Tongue River at State line, the flow-weighted average SC $(2,260 \mu \mathrm{S} / \mathrm{cm})$ of the $12 \mathrm{CBM}$ discharges measured in 2005 , and various rates of $\mathrm{CBM}$ discharge equivalent to multiples of the maximum allowable CBM discharge $(2,500 \mathrm{gal} / \mathrm{min}$, or $5.57 \mathrm{ft}^{3} / \mathrm{s}$ ) that was permitted at the time of the synoptic sampling trips. Specific conductance data for CBM discharges from the September 2005 synoptic investigation were used in these calculations because all the flowing CBM discharges were measured and more were sampled than in April 2006. The estimated historical and projected SC values determined by the calculations described in this paragraph are shown in figure 11.

The potential effects that direct CBM discharge might have on the SC of the Tongue River near the Tongue River Reservoir can be evaluated by examining and comparing the estimated historical and projected SC values for a range of streamflows (fig. 11). The projected SC values for flows of 50 to $3,000 \mathrm{ft}^{3} / \mathrm{s}$ in the Tongue River would not exceed the State of Montana irrigation-season standard of $1,500 \mu \mathrm{S} / \mathrm{cm}$ (Montana Department of Environmental Quality, 2006) for individual water samples (table 5) at CBM discharges as large as $5,000 \mathrm{gal} / \mathrm{min}$, a rate equivalent to twice the maximum allowable CBM discharge that was permitted at the time of the synoptic sampling trips (fig. 11). In addition, CBM discharge would have a larger effect on SC during lower flows than at higher flows (fig. 11). For example, at very low flow $\left(50 \mathrm{ft}^{3} / \mathrm{s}\right)$ with no CBM discharge, the estimated historical data indicate that $\mathrm{SC}$ would be $1,170 \mu \mathrm{S} / \mathrm{cm}$, whereas if streamflow was $50 \mathrm{ft}^{3} / \mathrm{s}$ and CBM discharges ranged from 1,250 to $5,000 \mathrm{gal} / \mathrm{min}$, the $\mathrm{SC}$ is projected to range from 1,230 to $1,370 \mu \mathrm{S} / \mathrm{cm}$, which represents an increase of 5.1 to 17 percent above the estimated historical SC. These projected $\mathrm{SC}$ values, as well as the estimated historical value without CBM discharge, exceed the State of Montana irrigation-season monthly mean standard of $1,000 \mu \mathrm{S} / \mathrm{cm}$ (table 5). Daily mean flows of $50 \mathrm{ft}^{3} / \mathrm{s}$ or less at Tongue River at State line occurred 120 times during the 39-year streamflow record (water years 1961-99) before CBM development but occurred 133 times during the 8-year period (water years 2000-2007) of CBM development (U.S. Geological Survey, 2007c). Severe drought conditions persisted during the 2004 and 2006 irrigation seasons (Montana Natural Resource Information System, 2008). If streamflow in the Tongue River near the Tongue River Reservoir was low $\left(100 \mathrm{ft}^{3} / \mathrm{s}\right)$ with no CBM discharge, the estimated historical data indicate that SC would be $871 \mu \mathrm{S} / \mathrm{cm}$, whereas if the streamflow was $100 \mathrm{ft}^{3} / \mathrm{s}$ and CBM discharges ranged from 1,250 to $5,000 \mathrm{gal} / \mathrm{min}$, the $\mathrm{SC}$ is projected to range from 909 to $1,010 \mu \mathrm{S} / \mathrm{cm}$, which represents an increase of 4.4 to 16 percent above the estimated historical SC. In comparison, if streamflow in the river was $600 \mathrm{ft}^{3} / \mathrm{s}$ with no $\mathrm{CBM}$ discharge, the estimated historical data indicate that $\mathrm{SC}$ would be $403 \mu \mathrm{S} / \mathrm{cm}$, whereas if the flow was $600 \mathrm{ft}^{3} / \mathrm{s}$ and CBM discharges ranged from 1,250 to $5,000 \mathrm{gal} / \mathrm{min}$, the SC is projected to range from 412 to $437 \mu \mathrm{S} / \mathrm{cm}$, which represents an increase of 2.2 to 8.4 percent above the estimated historical SC. Finally, at flows greater than $600 \mathrm{ft}^{3} / \mathrm{s}$, potential increases in $\mathrm{SC}$ are projected to be small (less than 8.4 percent).

The actual measured effect of CBM discharge on SC in the Tongue River was calculated by using the measured SC from the Tongue River and the CBM discharge outfalls, whereas the potential effects were projected by using regression-estimated historical SC data and mass-balance calculations for a range of flow conditions and CBM discharges. During both synoptic sampling trips, measured effects on SC from CBM discharges were similar to the projected effects. For example, on September 27, 2005, the instantaneous streamflow of the Tongue River at State line was $195 \mathrm{ft}^{3} / \mathrm{s}$ (river mile 30.57; table 1), and as noted in the section "Specific Conductance, September 2005," the estimated increase in $\mathrm{SC}$ of the Tongue River from CBM discharges was $27 \mu \mathrm{S} / \mathrm{cm}$, or 4.5 percent. In comparison, the projected increase in SC above the estimated historical SC is $25 \mu \mathrm{S} / \mathrm{cm}$, or 3.9 percent, for a streamflow of $195 \mathrm{ft}^{3} / \mathrm{s}$ and the measured CBM discharge of $3.12 \mathrm{ft}^{3} / \mathrm{s}(1,400 \mathrm{gal} / \mathrm{min})$.

The results from the April 2006 synoptic investigation also demonstrate that the projected $\mathrm{SC}$ values provide a reasonable measure of relative effect of $\mathrm{CBM}$ discharges on 


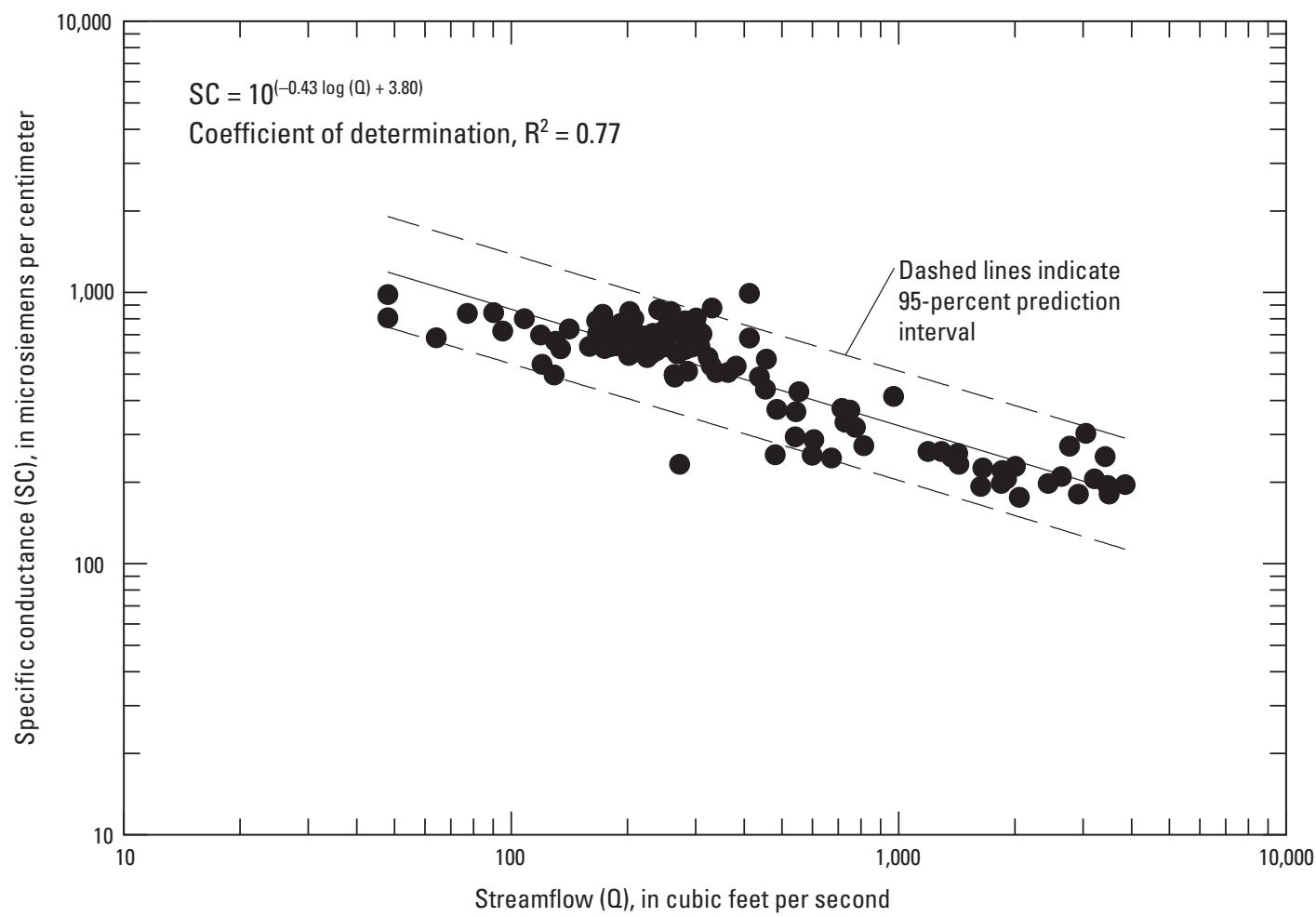

Figure 10. Relation between streamflow and measured specific conductance prior to coal-bed methane development, Tongue River at State line, near Decker, Mont. (06306300), water years 1985-99.

the Tongue River. For example, the instantaneous streamflow of the Tongue River near the Tongue River Reservoir on April 20, 2006, was $210 \mathrm{ft}^{3} / \mathrm{s}$ (as measured at river mile 32.81 ; table 3), and as noted in the section "Specific Conductance, April 2006," the increase in SC of the Tongue River from CBM discharges was $30 \mu \mathrm{S} / \mathrm{cm}$, or 5.8 percent. In comparison, the projected increase in $\mathrm{SC}$ above the estimated historical SC is $27 \mu \mathrm{S} / \mathrm{cm}$, or 4.3 percent, for a streamflow of $210 \mathrm{ft}^{3} / \mathrm{s}$ and the measured CBM discharge of $4.65 \mathrm{ft}^{3} / \mathrm{s}$ (2,090 gal/min).

This analysis of potential water-quality effects on the Tongue River assumes that the quantity and quality of water flowing into the study reach during the time of this study was the same as during the period before CBM development (data from water years 1985-99). Conditions in the upstream watersheds may have changed, but insufficient data exist to test this assumption. In addition, the estimated historical SC values for the predevelopment period are a general approximation based on regression analysis (Helsel and Hirsch, 2002), which has some uncertainty. This uncertainty can be represented by the 95-percent prediction interval shown in figure 10 and would increase the range in percent changes of projected SC in the Tongue River during various rates of CBM discharge (fig. 11). For example, if the estimated historical SC $(871 \mu \mathrm{S} / \mathrm{cm})$ is associated with a 20 -percent error, the range in percent changes in $\mathrm{SC}$ in the Tongue River at a streamflow of $100 \mathrm{ft}^{3} / \mathrm{s}$ would increase from a range of 4.4 to 16 percent to a range of 3.2 to 23 percent. In comparison, with an estimated historical SC of $403 \mu \mathrm{S} / \mathrm{cm}$ and the same 20-percent error, at $600 \mathrm{ft}^{3} / \mathrm{s}$, the range in percent changes in SC would increase from a range of 2.2 to 8.4 percent to a range of 1.7 to 11 percent. These results show that even moderately large errors in the estimated historical SC values have relatively little effect on the estimation of potential changes in water quality of the Tongue River that might be caused by CBM discharge.

\section{Sodium-Adsorption Ratio}

SAR is a water-quality characteristic used to classify the suitability of water for irrigation. The higher the SAR, the less suitable the water is for irrigation; SAR values greater than 4.5 to 7.5 are high, as indicated by State of Montana numeric standards (Montana Department of Environmental Quality, 2006) (table 5). SAR typically is determined by an equation using the laboratory-measured concentrations of sodium, calcium, and magnesium in a water sample:

$$
S A R=\frac{N a^{+}}{\sqrt{\frac{C a^{2+}+M g^{2+}}{2}}},
$$

where $\mathrm{Na}^{+}, \mathrm{Ca}^{2+}$, and $\mathrm{Mg}^{2+}$ are sodium, calcium, and magnesium concentrations, respectively, in milliequivalents per liter 


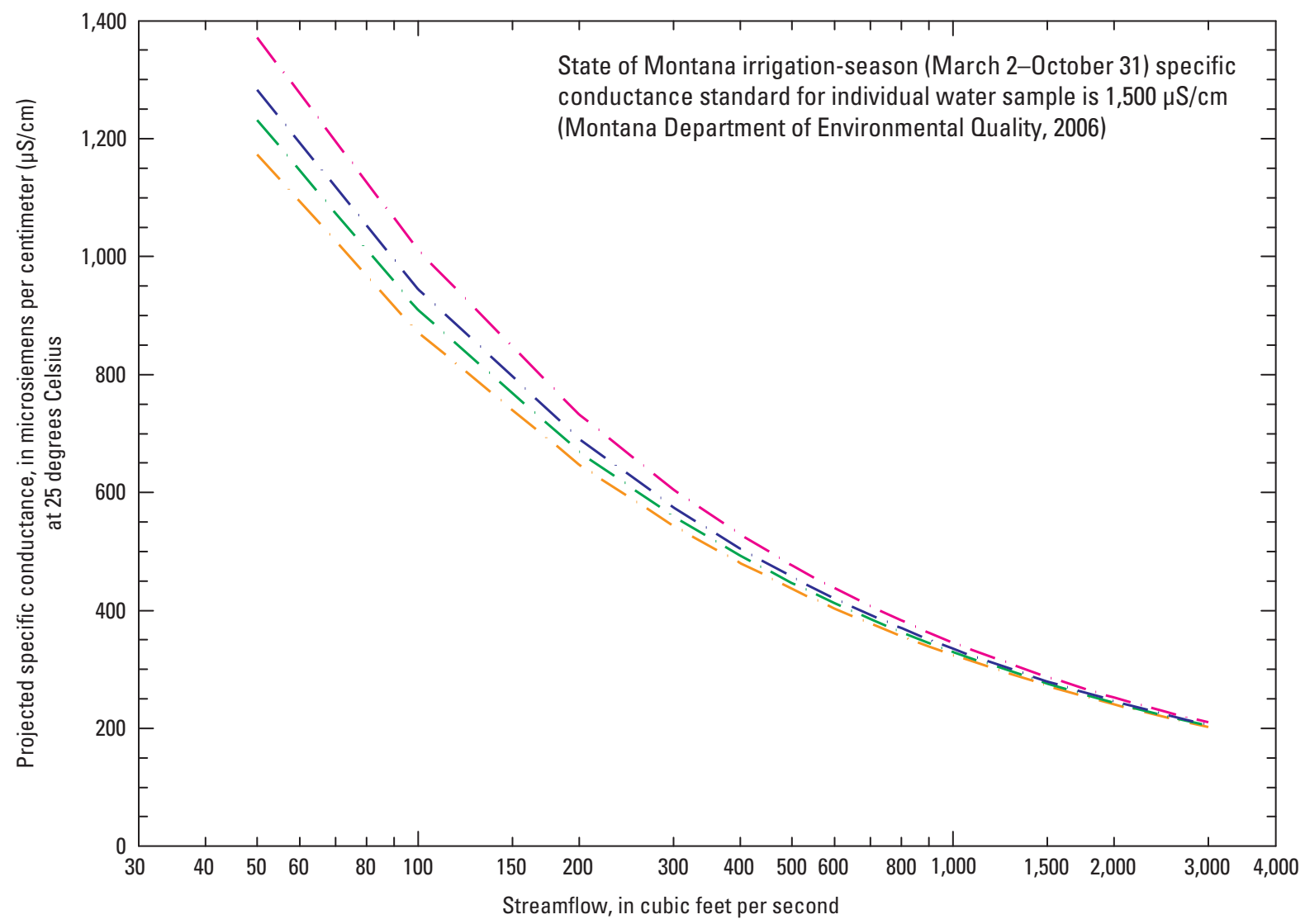

EXPLANATION

Projected specific conductance

_- Twice maximum allowable coal-bed methane discharge permitted at time of sampling $(5,000$ gallons per minute $)$
- - Maximum allowable coal-bed methane discharge permitted at time of sampling $(2,500$ gallons per minute $)$
- $-\quad$ One-half maximum allowable coal-bed methane discharge permitted at time of sampling $(1,250$ gallons per minute $)$
No coal-bed methane discharge, from regression equation for historical data (fig. 10)

Figure 11. Relation between streamflow and projected specific conductance at Tongue River near the Tongue River Reservoir, Mont., for various rates of coal-bed methane discharge.

Table 5. State of Montana numeric standards for specific conductance and sodium-adsorption ratio.

[Montana Department of Environmental Quality (2006). Abbrevation: $\mu \mathrm{S} / \mathrm{cm}$, microsiemens per centimeter at 25 degrees Celsius]

\begin{tabular}{|c|c|c|c|c|}
\hline \multirow{2}{*}{ Season } & \multicolumn{2}{|c|}{$\begin{array}{c}\text { Specific conductance } \\
(\mu \mathrm{S} / \mathrm{cm})\end{array}$} & \multicolumn{2}{|c|}{ Sodium-adsorption ratio } \\
\hline & $\begin{array}{c}\text { Individual water } \\
\text { sample }\end{array}$ & Monthly mean & $\begin{array}{c}\text { Individual water } \\
\text { sample }\end{array}$ & Monthly mean \\
\hline Irrigation season (March 2-October 31) & 1,500 & 1,000 & 4.5 & 3.0 \\
\hline Nonirrigation season (November 1-March 1) & 2,500 & 1,500 & 7.5 & 5.0 \\
\hline
\end{tabular}


(Hem, 1985). SAR also can be estimated from SC by a regression relation between SC and SAR for sites that have been previously sampled and for which the relation is statistically significant (Cannon and others, 2007).

SAR was calculated for the water-quality samples collected during both synoptic investigations. In September 2005, SAR values for the five sampled CBM discharges ranged from 42 to 58 and were 48 to 161 times greater than the estimated SAR values for the Tongue River and tributary sites with continuous estimated SAR (tables 1 and 2). In April 2006, SAR values for the three sampled CBM discharges ranged from 46 to 59 and were 35 to 179 times greater than the estimated SAR values determined for the Tongue River and tributary sites with continuous estimated SAR (tables 3 and 4).

With SAR values as much as two orders of magnitude larger than those in the Tongue River, the CBM discharges had the potential to increase measurably the SAR in the Tongue River. Estimates of potential effects of the CBM discharges on the SAR of the Tongue River near the Tongue River Reservoir were calculated using a multistep process involving linear regression and mass-balance calculations for sodium, calcium, and magnesium (the SAR ions) for a range of streamflow and CBM-discharge conditions. The first step in the calculation process was to determine the historical concentrations of each SAR ion for a given streamflow prior to CBM development. Data were retrieved (U.S. Geological Survey, 2007c) for samples collected during water years 1985-99 at Tongue River at State line and used to develop regression equations to estimate historical concentrations of these ions for a given streamflow (fig. 12). In addition, estimated historical SAR values were calculated from the estimated historical ion concentrations using equation 3 for a range of streamflows. The second step involved calculating the flow-weighted average concentration of each SAR ion in the measured CBM discharge. These concentrations were calculated from CBM discharge data from September 2005 (table 2) because all the flowing CBM discharges were measured and more were sampled than in April 2006. In the third step, potential increases in the concentrations of each SAR ion in the Tongue River near the Tongue River Reservoir resulting from CBM discharge were projected using mass-balance calculations (equations 1 and 2) for a range of streamflows and CBM discharges. Data used in the calculations included the calculated estimated historical concentrations of the SAR ions, a range of streamflows (50 to $3,000 \mathrm{ft}^{3} / \mathrm{s}$ ) representative of most historical flow conditions for Tongue River at State line, the flow-weighted average concentration of each SAR ion in the five CBM discharges sampled in 2005 (table 2), and various rates of CBM discharge equivalent to multiples of the maximum allowable CBM discharge $\left(2,500 \mathrm{gal} / \mathrm{min}\right.$, or $\left.5.57 \mathrm{ft}^{3} / \mathrm{s}\right)$ that was permitted at the time of the synoptic sampling trips. In the final step, the projected SAR values for the Tongue River near the Tongue River Reservoir were calculated from the projected concentrations of each SAR ion using equation 3 . The estimated historical and projected SAR values determined by the calculations described in this paragraph are shown in figure 13.
The potential effects that direct CBM discharge might have on the SAR of the Tongue River near the Tongue River Reservoir can be evaluated by examining and comparing the estimated historical and projected SAR values for a range of streamflows (fig. 13). The projected SAR values in the Tongue River near the Tongue River Reservoir for flows of 50 to 3,000 ft $\mathrm{ft}^{3} / \mathrm{s}$ would not exceed the State of Montana irrigation-season standard of 4.5 (Montana Department of Environmental Quality, 2006) for individual water samples (table 5) at CBM discharges as large as 5,000 gal/min, a rate equivalent to twice the maximum allowable CBM discharge that was permitted at the time of the synoptic sampling trips (fig. 13). Similar to SC, CBM discharge would have a larger effect on the SAR during lower flows than during higher flows (fig. 13), but the percent increases would be larger for the SAR than for SC. For example, at very low flow $\left(50 \mathrm{ft}^{3} / \mathrm{s}\right)$ with no CBM discharge, the estimated historical SAR would be 1.06, whereas if streamflow was $50 \mathrm{ft}^{3} / \mathrm{s}$ and CBM discharges ranged from 1,250 to $5,000 \mathrm{gal} / \mathrm{min}$, the SAR is projected to range from 1.60 to 3.05 , or 51 to 188 percent greater than the estimated historical SAR. If very low flows were sustained, the State of Montana irrigation-season monthly mean standard of 3.0 (table 5) might be exceeded at CBM discharges of about $5,000 \mathrm{gal} / \mathrm{min}$. If streamflow in the Tongue River near the Tongue River Reservoir was $100 \mathrm{ft}^{3} / \mathrm{s}$ with no CBM discharge, the estimated historical SAR would be 0.83 , whereas if the streamflow was $100 \mathrm{ft}^{3} / \mathrm{s}$ and CBM discharges ranged from 1,250 to $5,000 \mathrm{gal} / \mathrm{min}$, the SAR is projected to range from 1.15 to 2.08 , which represents a 39 to 151 percent increase above the estimated historical SAR. In comparison, if streamflow in the river was $600 \mathrm{ft}^{3} / \mathrm{s}$ with no CBM discharge, the estimated historical SAR would be 0.43 , whereas if the streamflow was $600 \mathrm{ft}^{3} / \mathrm{s}$ and CBM discharges ranged from 1,250 to $5,000 \mathrm{gal} / \mathrm{min}$, the SAR is projected to range from 0.52 to 0.77 , which represents a 21 to 79 percent increase. Finally, if the streamflow in the river was $3,000 \mathrm{ft}^{3} / \mathrm{s}$ and CBM discharges ranged from 1,250 to $5,000 \mathrm{gal} / \mathrm{min}$, the SAR is projected to range from 0.26 to 0.34 , which represents a small but measurable increase ( 8 to 42 percent) above the estimated historical SAR of 0.24 .

During the September 2005 synoptic investigation, the measured effect of CBM discharges permitted at the time of sampling, the SAR in the Tongue River was similar to the projected effect. For example, on September 27, 2005, when streamflow at Tongue River at State line was $195 \mathrm{ft}^{3} / \mathrm{s}$, the measured SAR was 0.76 (table 1). The estimated historical SAR (fig. 13) would be 0.65 for the same streamflow; thus, the measured SAR was 0.11 higher than the estimated historical SAR. In comparison, for a streamflow in the Tongue River near the Tongue River Reservoir of $195 \mathrm{ft}^{3} / \mathrm{s}$ and the combined CBM discharge of $3.12 \mathrm{ft}^{3} / \mathrm{s}$ (measured in September 2005), the projected SAR is 0.87 , which represents an increase of 0.22 above the estimated historical SAR of 0.65 . Although the projected increase in the SAR $(0.22)$ is twice the measured increase (0.11) above the estimated historical value, the magnitudes of these SAR differences are small and indicate that 
Figure 12. Relation between streamflow and $A$, sodium; $B$, calcium; and $C$, magnesium concentrations from water-quality samples collected prior to coal-bed methane development, Tongue River at State line, near Decker, Mont. (06306300), water years 1985-99, used to estimate sodium-adsorption ratio (SAR) for various streamflows in figure 13.
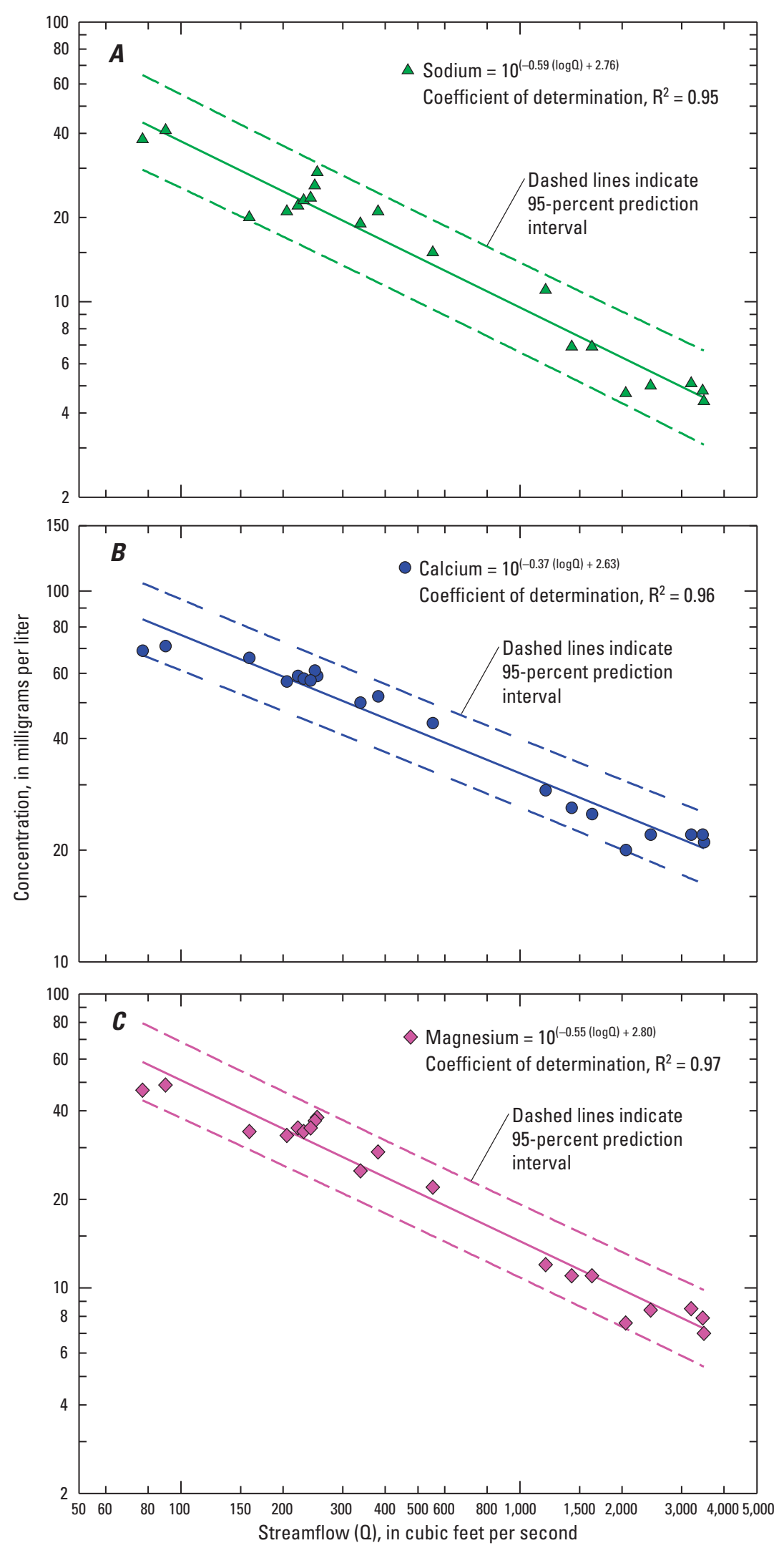


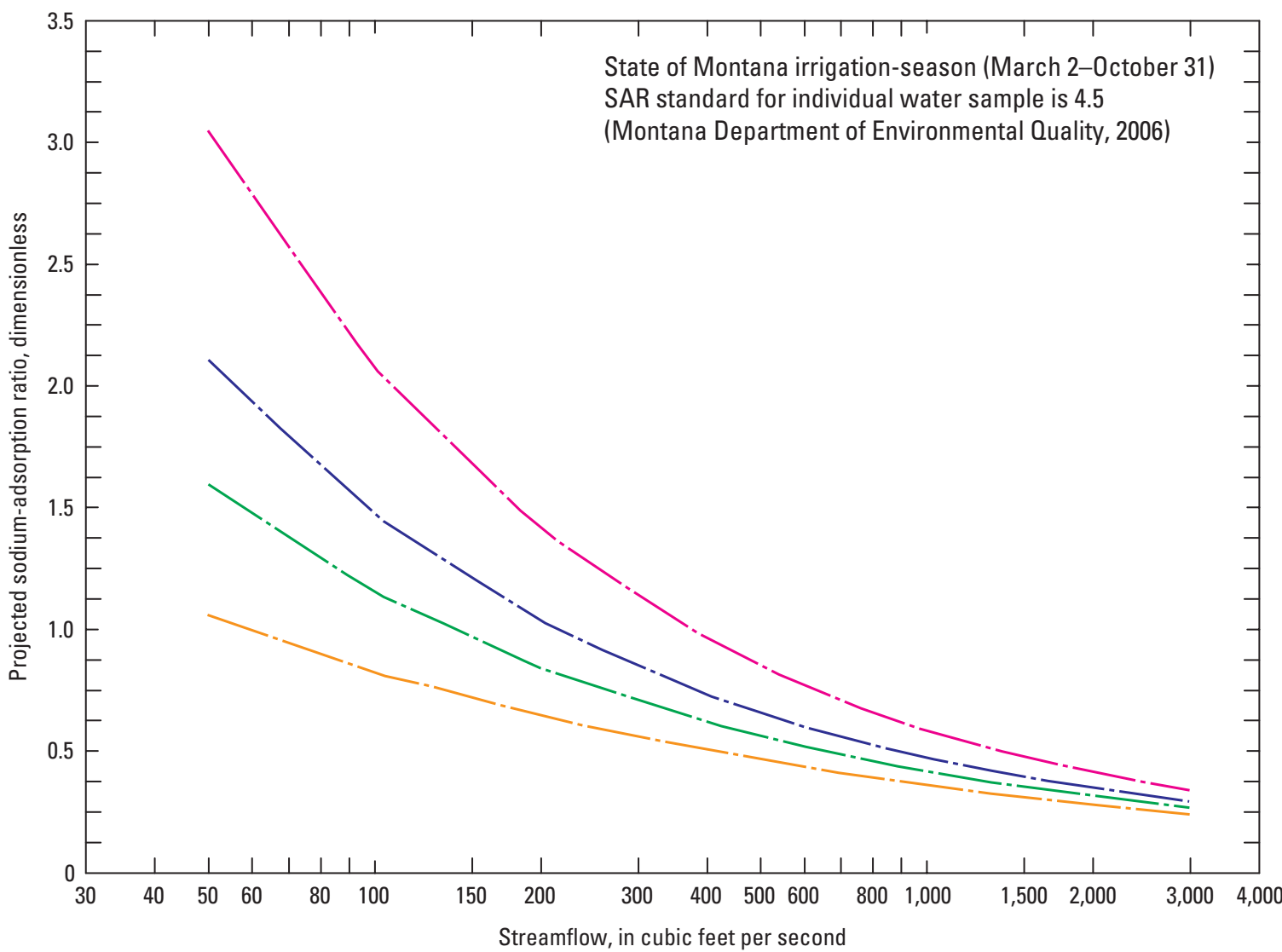

EXPLANATION

Projected sodium-adsorption ratio (SAR)

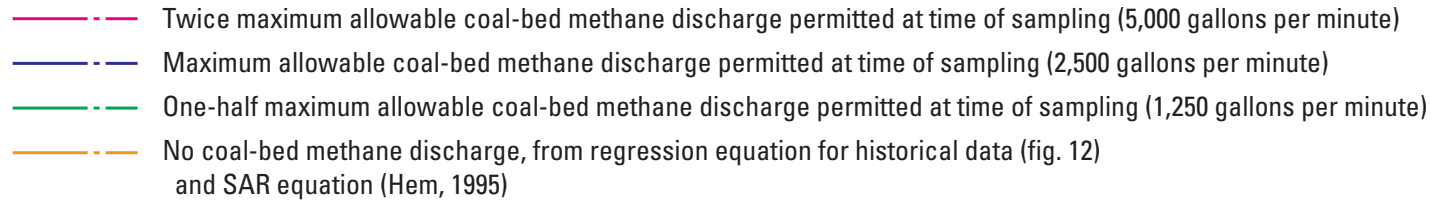

Figure 13. Relation between streamflow and projected sodium-adsorption ratio at Tongue River near the Tongue River Reservoir, Mont., for various rates of coal-bed methane discharge.

the projected values provide a reasonable measure for evaluating potential water-quality effects from CBM discharges. The measured and projected SAR for the April 2006 synoptic investigation could not be compared because the SAR of the river was not measured at the downstream end of the study reach.

Similar to the projection of potential SC values resulting from CBM discharge, the projections of SAR assume that the quantity and quality of water flowing into the study reach during the time of this study was the same as during the period before CBM development (water years 1985-99). In addition, the projected SAR values shown in figure 13 have some uncertainty because the historical concentrations for each of the SAR ions (fig. 12) are general approximations based on regression analysis (Helsel and Hirsch, 2002). This uncertainty can be represented by the 95-percent prediction intervals shown in figure 12 and would increase the range in percent changes of projected SAR in the Tongue River during various rates of CBM discharge (fig. 13). For example, if the estimated historical SAR (0.83) is associated with a 20-percent error, the range in percent changes in SAR in the Tongue River at a streamflow of $100 \mathrm{ft}^{3} / \mathrm{s}$ would increase from a range of 39 to 151 percent to a range of 33 to 159 percent. In comparison, for the same 20-percent error at $600 \mathrm{ft}^{3} / \mathrm{s}$, the range in percent changes in SAR would increase from a range of 21 to 79 percent to a range of 11 to 80 percent. These results show that even moderately large errors in the estimated historical values have relatively little effect on the projection of potential changes in water quality of the Tongue River that might be caused by CBM discharges. 


\section{Summary and Conclusions}

The purpose of this report is to describe the downstream changes in water quality along the upper Tongue River between Monarch, Wyoming, and the Tongue River Reservoir in Montana as indicated by the results of two synoptic sampling trips and to estimate the potential water-quality effects of directly discharging coal-bed methane (CBM) production water (CBM discharge) in the river in this reach. CBM discharge could potentially increase the salinity (as measured by specific conductance) and sodium-adsorption ratio (SAR) in the river. Two synoptic sampling trips were conducted in September 2005 and April 2006 to assess these potential effects.

The study area is the reach of the upper Tongue River extending from the gaging station at Monarch (gaging station 06299980) downstream to the Otter Road bridge, which is just upstream from the Tongue River Reservoir. Streamflow and specific conductance (SC) were measured at multiple locations on the Tongue River throughout the study reach and for most inflows. Continuous streamflow data were available from two gaging stations on the Tongue River and gaging stations on two major tributary streams. Water-quality samples for SAR determination were collected at selected inflow sites.

SC generally increased in the downstream direction during both synoptic sampling trips. Large increases in SC occurred downstream from the two main tributaries: Goose Creek (16 to 24 percent) and Prairie Dog Creek (12 to 15 percent). During the September 2005 synoptic sampling, the estimated increase in SC of the Tongue River at State line resulting from all $15 \mathrm{CBM}$ discharge outfalls was $27 \mu \mathrm{S} / \mathrm{cm}$ (an increase of 4.5 percent). Of that $27-\mu \mathrm{S} / \mathrm{cm}$ increase, about $17 \mu \mathrm{S} / \mathrm{cm}$ can be attributed to the large flow from CBM outfall MT-0030457-15 downstream from Prairie Dog Creek. During the April 2006 synoptic sampling, the discharge from CBM outfall MT-0030457-16 likely caused the $30-\mu \mathrm{S} / \mathrm{cm}$ increase in $\mathrm{SC}$ in the main stem between river miles 31.74 and 32.81 downstream of Tongue River at State line.

Estimates of potential effects of the CBM discharges on the SC of the Tongue River near the Tongue River Reservoir were calculated using a two-step process involving linear regression and mass-balance calculations for a range of streamflow and CBM-discharge conditions. Results from the calculations indicate that projected SC and SAR values in the Tongue River near the Tongue River Reservoir for streamflows of 50 to $3,000 \mathrm{ft}^{3} / \mathrm{s}$ would not exceed the State of Montana irrigation-season standards for SC and SAR for individual water samples at CBM discharges as large as 5,000 gal $/ \mathrm{min}$, a rate equivalent to twice the maximum allowable CBM discharge that was permitted at the time of the synoptic sampling trips. Also, the effects from CBM discharge are projected to be larger at lower flows than at higher flows. For example, the $\mathrm{SC}$ is projected to increase 4.4 to 16 percent above estimated historical SC if the streamflow was $100 \mathrm{ft}^{3} / \mathrm{s}$ in the Tongue River near the Tongue River Reservoir and CBM discharge ranged from 1,250 to $5,000 \mathrm{gal} / \mathrm{min}$. In comparison, the $\mathrm{SC}$ is projected to increase only 2.2 to 8.4 percent if the streamflow was $600 \mathrm{ft}^{3} / \mathrm{s}$ and CBM discharge ranged from 1,250 to 5,000 $\mathrm{gal} / \mathrm{min}$. During both synoptic investigations, the measured effect of CBM discharge on SC was similar to the projected effect. For example, on September 27, 2005, the increase in SC calculated from measured data was 4.5 percent, whereas, the projected increase is 3.9 percent. On April 20, 2006, the actual measured increase was 5.8 percent, whereas the projected increase is 4.3 percent.

In September 2005, SAR values for the five sampled CBM discharges ranged from 42 to 58 and were 48 to 161 times greater than the estimated SAR values for the Tongue River and tributary sites with continuous estimated SAR. In April 2006, SAR values for the three sampled CBM discharges ranged from 46 to 59 and were 35 to 179 times greater than the estimated SAR values determined for the Tongue River and tributary sites with continuous estimated SAR. The high SAR values of the CBM discharges indicate a potential to increase the SAR in the Tongue River.

Similar to SC, SAR at Tongue River near the Tongue River Reservoir would be affected more by CBM discharges at lower flows than at higher flows. For example, the SAR is projected to range from 39 to 151 percent above the estimated historical SAR if the streamflow was $100 \mathrm{ft}^{3} / \mathrm{s}$ and CBM discharge ranged from 1,250 to 5,000 gal/min. In comparison, the SAR is projected to increase only 21 to 79 percent if the streamflow was $600 \mathrm{ft}^{3} / \mathrm{s}$ and CBM discharge ranged from 1,250 to $5,000 \mathrm{gal} / \mathrm{min}$.

During the September 2005 synoptic investigation, the measured effect of CBM discharges on SAR in the Tongue River at State line was similar to the projected effect. For example, on September 27, 2005, the increase in SAR calculated from measured data was 0.11 , whereas, the projected increase is 0.22 . Although the projected increase in SAR is twice the measured increase, the magnitudes of these SAR differences are small and indicate that the estimates of SAR provide a reasonable relative measure for evaluating potential water-quality effects from CBM discharges. A comparison of the measured and projected SAR values for the April 2006 synoptic investigation could not made because the SAR of the river was not measured at the downstream end of the study reach.

This analysis of potential water-quality effects on the SC and SAR of the Tongue River in the study area assumes that the quantity and quality of water flowing into the study reach at the time of this study was the same as during the period before CBM development (data from water years 1985-99). Conditions in the upstream watersheds may have changed, but insufficient data exist to test this assumption. 


\section{References Cited}

Berkas, W.R., White, M.K., Ladd, P.B., Bailey, F.A., and Dodge, K.A., 2005, Water resources data, Montana, water year 2004, v. 2, Yellowstone and upper Columbia River basins and ground-water levels: U.S. Geological Survey Water-Data Report MT-04-2, 555 p.

Berkas, W.R., White, M.K., Ladd, P.B., Bailey, F.A., and Dodge, K.A., 2006, Water resources data, Montana, water year 2005, v. 2, Yellowstone and upper Columbia River basins and ground-water levels: U.S. Geological Survey Water-Data Report MT-05-2, 561 p.

Cannon, M.R., Nimick, D.A., Cleasby, T.E., Kinsey, S.M., and Lambing, J.H., 2007, Measured and estimated sodiumadsorption ratios for Tongue River and its tributaries, Montana and Wyoming, 2004-06: U.S. Geological Survey Scientific Investigations Report 2007-5072, 45 p. (Only available at http://pubs.usgs.gov.sir.2007/5072/.)

Clark, M.L., and Mason, J.P., 2007, Water-quality characteristics for sites in the Tongue, Powder, Cheyenne, and Belle Fourche River drainage basins, Wyoming and Montana, water years 2001-2005, with temporal patterns of selected long-term water-quality data: U.S. Geological Survey Scientific Investigations Report 2007-5146, 65 p. (Also available online at http://pubs.usgs.gov/sir/2007/5146/.)

Edwards, T.K., and Glysson, G.D., 1999, Field methods for measurement of fluvial sediment: U.S. Geological Survey Techniques of Water-Resources Investigations, book 3, chap. C2, 89 p. (Also available online at http://pubs.usgs. gov/twri/.)

Fishman, M.J., ed., 1993, Methods of analysis by the U.S. Geological Survey National Water Quality LaboratoryDetermination of inorganic and organic constituents in water and fluvial sediments: U.S. Geological Survey OpenFile Report 93-125, 217 p.

Friedman, L.C., and Erdmann, D.E., 1982, Quality assurance practices for the chemical and biological analyses of water and fluvial sediments: U.S. Geological Survey Techniques of Water-Resources Investigations, book 5, chap. A6, $181 \mathrm{p}$. (Also available online at http://pubs.usgs.gov/twri/.)

Helsel, D.R., and R.M. Hirsch, 2002, Statistical methods in water resources: U.S. Geological Survey Techniques of Water-Resources Investigations, book 4, chap. A3, 522 p. (Also available at http://pubs.usgs.gov/twri/.)

Hem, J.D., 1985, Study and interpretation of the chemical characteristics of natural water (3rd ed.): U.S. Geological Survey Water-Supply Paper 2254, 264 p.
Horowitz, A.J., Demas, C.R., Fitzgerald, K.K., Miller, T.L., and Rickert, D.A., 1994, U.S. Geological Survey protocol for the collection and processing of surface-water samples for the subsequent determination of inorganic constituents in filtered water: U.S. Geological Survey Open-File Report 94-539, 57 p.

Jones, B.E., 1987, Quality control manual of the U.S. Geological Survey's National Water Quality Laboratory: U.S. Geological Survey Open-File Report 87-457, 17 p.

Keith, Kristin, Bauder, James, and Wheaton, J.R., 2003, Frequently asked questions, coal bed methane: MSU Extension Water Quality Program, Coal bed methane, accessed August 17, 2010, at http://waterquality.montana.edu/docs/ methane/cbmfaq.shtml.

Lambing, J.H., comp., 2006, Quality-assurance plan for waterquality activities of the U.S. Geological Survey Montana Water Science Center: U.S. Geological Survey Open-File Report 2006-1275, 39 p.

Montana Department of Environmental Quality, 2006, Administrative Rules of Montana, Water Quality, Surface Water Quality Standards and Procedures, Rule 17.30.670, Numeric Standards for Electrical Conductivity (EC) and Sodium Adsorption Ratio (SAR), accessed August 13, 2010, at http://www.mtrules.org/gateway/ruleno. asp? $R N=17 \% 2 E 30 \% 2 E 670$.

Montana Natural Resource Information System, 2008, Montana county drought status for 2004-08 [by month], accessed June 27, 2008, at http://nris.state.mt.us/drought/ status/status2008.html.

Montana Supreme Court, 2010, State Law Library of Montana-Montana Supreme Court Cases [Case number DA 09-0131], 19 p., accessed online January 6, 2011, at http:// searchcourts.mt.gov/.

National Oceanic and Atmospheric Administration, 2007, Precipitation amounts for September 2005 and April 2006 at Sheridan [Wyoming], accessed October 25, 2007, at http:// www.wrh.noaa.gov/byz/unique_climate.php.

Orion Research, Inc., 1990, Model 124 Conductivity/TDS, Hand-Held Set, Meter, Instruction manual: Boston, Mass., Orion Research, Inc., 13 p.

Pritt, J.W., and Raese, J.W., eds., 1995, Quality assurance/ quality control manual-National Water Quality Laboratory: U.S. Geological Survey Open-File Report 95-443, $35 \mathrm{p}$.

Rantz, S.E., and others, 1982, Measurement and computation of streamflow: U.S. Geological Survey Water-Supply Paper 2175, 2 v., 631 p. 
Rice, C.A., Ellis, M.S., and Bullock, J.H., Jr., 2000, Water coproduced with coalbed methane in the Powder River Basin, Wyoming; preliminary compositional data: U.S. Geological Survey Open-File Report 00-372, 20 p.

U.S. Department of Agriculture, 1995, Soil survey laboratory information manual: Natural Resource Conservation Service, Soil Survey Laboratory Investigation Report No. 45, version 1.0, 316 p., accessed June 26, 2008, at http://ftp-fc. sc.egov.usda.gov/NSSC/Lab_info_manual.ssir45.pdf.

U.S. Geological Survey, 2007a, Annual mean streamflow at Goose Creek near Acme [Wyoming] (06305700), accessed April 9, 2008, at http://waterdata.usgs.gov/nwis.

U.S. Geological Survey, 2007b, Annual mean streamflow at Prairie Dog Creek near Acme [Wyoming] (06306250), accessed April 9, 2008, at http://waterdata.usgs.gov/nwis.

U.S. Geological Survey, 2007c, Annual mean streamflow at Tongue River at State line [Montana] (06306300), accessed April 9, 2008, at http://waterdata.usgs.gov/nwis.

U.S. Geological Survey, 2007d, Water-resources data for the United States, water year 2006: U.S. Geological Survey Water-Data Report WDR-US-2006. (Also available online at http://pubs.water.usgs.gov/wdr2006.)

U.S. Geological Survey, variously dated, National field manual for the collection of water-quality data: U.S. Geological Survey Techniques of Water-Resources Investigations, book 9, chaps. A1-A9. (Also available online at http://pubs. usgs.gov/twri/.)
Van Voast, W.A., 2003, Geochemical signature of formation waters associated with coalbed methane: American Association of Petroleum Geologists, v. 87, no. 4, p. 667-676.

Ward, J.R., and Harr, C.A., eds., 1990, Methods for collection and processing of surface-water and bed-material samples for physical and chemical analyses: U.S. Geological Survey Open-File Report 90-140, 71 p.

Wyoming Oil and Gas Commission, 2008a, Production for Badger Creek drainage, accessed June 26, 2008, at http:// wogcc.state.wy.us/crmsprod.cfm? code $=7301$.

Wyoming Oil and Gas Commission, 2008b, Production for Prairie Dog Creek drainage, accessed June 26, 2008, at http://wogcc.state.wy.us/crmsprod.cfm? code $=7101$.

Wyoming Oil and Gas Commission, 2008c, Production for Upper Tongue River, accessed October 19, 2009, at http:// wogcc.state.wy.us/inddrainage.cfm? dcode $=1$.

YSI, Incorporated, 2004, YSI 556 Multi-Probe System (MPS) operations manual, accessed March 1, 2010, at http://www. ysi.com/media/pdfs/655279-YSI-556-Operations-ManualRevC.pdf.

\footnotetext{
Publishing support provided by the: Denver and Rolla Publishing Service Centers

\author{
For more information concerning this publication, contact: \\ Director, Montana Water Science Center \\ U.S. Geological Survey \\ 3162 Bozeman Ave. \\ Helena, MT 59601 \\ (406) 457-5900
}

Or visit the Montana Water Science Center Web site at: http://mt.water.usgs.gov/ 

